RICE UNIVERSITY

\title{
Reward Scheduling for QoS in Cloud Applications
}

by

Ahmed Abdelrahman Elnably

A Thesis Submitted

in Partial Fulfillment of the

REQUIREMENTS FOR THE DEGREE

Master of Science

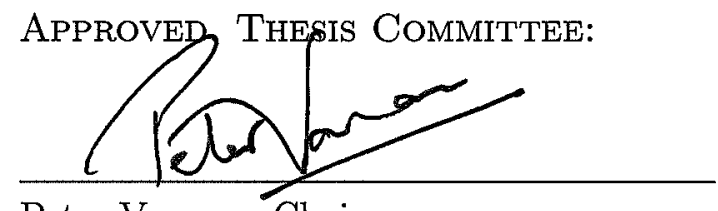

Peter Varman, Chair

Professor of Electrical and Computer

Engineering and Computer Science

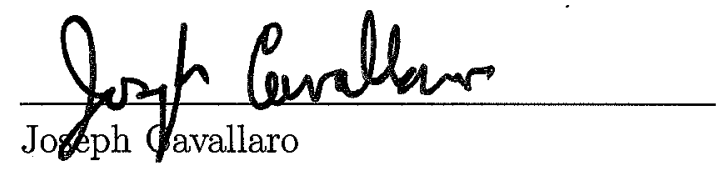

Professor of Electrical and Computer

Engineering and Computer Science

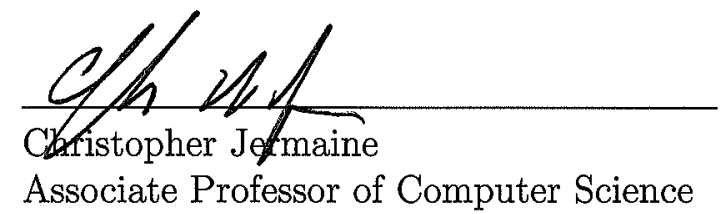

Houston, Texas

April, 2012 

To my family, the reason I stand where $I$ do 


\begin{abstract}
Reward Scheduling for QoS in Cloud Applications
\end{abstract}

by

\begin{abstract}
Ahmed Abdelrahman Elnably
The growing popularity of multi-tenant, cloud-based computing platforms is increasing interest in resource allocation models that permit flexible sharing of the underlying infrastructure. This thesis introduces a novel IO resource allocation model that better captures the requirements of paying tenants sharing a physical infrastructure. The model addresses a major concern regarding application performance stability when clients migrate from a dedicated to a shared platform. Specifically, while clients would like their applications to behave similarly in both situations, traditional models of fairness, like proportional share allocation, do not exhibit this behavior in the context of modern multi-tiered storage architectures.

We also present a scheduling algorithm, the Reward Scheduler, that implements the new allocation policy, by rewarding clients with better runtime characteristics, resulting in benefits to both the clients and the service provider. Moreover, the Reward scheduler also supports weight-based capacity allocation subject to a minimum reservation and maximum limitation on the IO allocation for each task. Experimental results indicate that the proposed algorithm proportionally allocates the system capacity in proportion to their entitlements.
\end{abstract}




\section{Acknowledgments}

First and foremost I owe my deepest gratitude to my advisor, Dr. Peter Varman, for his support, encouragement and patient guidance. Peter has been an excellent advisor, teacher and friend, he has provided the best possible research environment one could ask for. I would also like to express my gratitude to the members of my committee, Dr. Cavallaro and Dr. Jermaine, for their time and effort in reviewing my work. I wish to thank my friends Ahmed Maamoun, Murtada Al-Habib, Omar Oraby, Ahmad Hammoudi and all my dear friends, for their moral support and being true friends whom I can turn to when I need them. Also, this work would have never been possible without the assistance and advice of my student colleagues Kai Du and Hui Wang. Lastly, and most importantly, I wish to thank my family, my father Abdelrahman Elnably, my mother Sania Elshaaer for raising me, and my dear brother Architect Mahmoud Elnably. To them I dedicate this work. 


\section{Contents}

Abstract iv

Acknowledgments $\quad$ V

List of Illustrations viii

List of Tables $\quad$ X

1 Introduction 1

2 Related Work 4

2.1 Proportional Share Schedulers For Networks and CPU . . . . . . . . 4

2.2 Proportional Share Schedulers For IO and Disk Scheduling . . . . . . 4

2.3 Time-Quanta Based IO Allocation . . . . . . . . . . . . . . 5

2.4 Multi-Tag Schemes . . . . . . . . . . . . . . . 6

3 Reward Scheduling $\quad 8$

4 Reward Allocation Policy in Sequential Model 11

4.1 Reward Scheduling Algorithm . . . . . . . . . . . . . . . . 14

4.2 Experimental Evaluation . . . . . . . . . . . . . . . . 18

4.2.1 Guaranteeing Weight Proportional Allocation . . . . . . . . . 18

4.2 .2 Reward Scheduling . . . . . . . . . . . . . . . . . . . . 19

4.3 Summary ............................ 24

5 Reward Allocation Policy in Concurrent Model 25

5.1 Reward Scheduling Algorithm . . . . . . . . . . . . . . . . . 30 
5.2 Experimental Evaluation . . . . . . . . . . . . . . . . 33

5.3 Summary . . . . . . . . . . . . . . . . . 37

6 Reservations and Limits 39

6.1 Reward Scheduler with Reservations and Limits . . . . . . . . . . . . 41

6.2 Experimental Evaluation . . . . . . . . . . . . . . . . . 42

6.3 Summary .............................. 45

7 Conclusion $\quad 48$

Bibliography $\quad 49$ 


\section{Illustrations}

3.1 Model for Sequential Storage . . . . . . . . . . . . . . . . 9

3.2 Model for Multi-Tiered Storage . . . . . . . . . . . . . . . . . 10

4.1 Requests Service Pattern using PS. . . . . . . . . . . . . . 17

4.2 Requests Service Pattern using Reward Scheduling. . . . . . . . . . . 18

4.3 Guaranteeing Weight Proportional Allocation . . . . . . . . . . . . . 19

4.4 Comparison of Capacity Allocation in PS and Reward Schedulers . . 21

4.5 A and B run separately. Throughput is the clients entitlement. . . . . 22

4.6 Comparison of allocation when clients are run separately using PS and RAP. PS does not distinguish the behavior of the clients within a region, while the allocation by RAS is proportional to the clients' entitlements. Overall system throughput under RAP exceeds that under PS. . . . . . . . . . . . . . . . . . . . . . . . 23

4.7 Reward Scheduling with Hit Ratio. . . . . . . . . . . . . . . . . . 24

5.1 Throughput vs. Hit ratio, $\alpha=2 / 3 \ldots \ldots$. . . . . . . . 27

5.2 Throughput vs. Hit ratio, $\alpha=5 / 6 \ldots \ldots . \ldots 28$

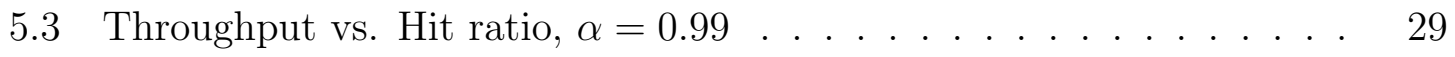

5.4 Requests Service Pattern using PS. . . . . . . . . . . . . . . . 33

5.5 Requests Service Pattern using Reward Scheduling. . . . . . . . . . . 33

5.6 Comparison of allocation while using Hit Ratio as a scaling parameter. 35

5.7 Device Utilizations. . . . . . . . . . . . . . . . . . . . . 36 
5.8 Reward Scheduling with Arbitrary Weights. . . . . . . . . . . . 36

5.9 Comparison of allocation when clients have different read/write ratios, with different queue lengths. . . . . . . . . . . . . . 38

6.1 Comparison Between Capacity Allocation With and Without rTag/wTag Synchronization . . . . . . . . . . . . . . . 46

6.2 Reward Scheduling with Reservations in Parallel Model . . . . . . . . 47 


\section{Tables}

4.1 Throughput Allocations under Reward Scheduler and PS. . . . . . . 13

4.2 Symbols used by the Scheduler . . . . . . . . . . . . . . . . . 17

5.1 Symbols used by the Scheduler . . . . . . . . . . . . . . . . . 31

6.1 Additional symbols used by the Reward Scheduler . . . . . . . . . . . 42 


\section{Chapter 1}

\section{Introduction}

The popularity of the cloud as a general-purpose computing platform is raising awareness of the need for versatile QoS policies and mechanisms that can support diverse applications and time-varying workloads [1]. Commercial virtualization technology $[2,3,4]$ has facilitated aggressive resource consolidation, whereby multiple tenants or clients can share computing, storage, and networking infrastructure provided by the service provider $[5,6,7]$. Customers benefit from ease and speed of deployment, elastic resource availability, and lowered costs for short-term or unknown computing needs, while service providers can reap the benefits of consolidation in terms of reduced infrastructure and management costs.

Despite the benefits of shared infrastructure and pay-as-you-go pricing models over dedicated and owned resources, performance concerns persist, especially in the area of QoS for shared storage and IO. Resource controls for storage servers [8, 9] are

still in an early stage of deployment [10], and provide relatively modest functionality. Unlike CPU and memory, storage resource management has to deal with stateful devices and variable service times, which make it much more difficult to provide effective QoS guarantees. The problem will increase as new multi-tiered storage organizations supplant traditional hard-disk SAN and NAS arrays. By employing aggressive SSDbased tiering or caching within a traditional SAN or distributed clustered storage [11], these solutions boost performance at a reasonable cost. As a consequence, QoS management now needs to deal with multiple heterogeneous devices with very different 
access speeds, and applications whose performance changes drastically with changes in the access profiles. In Chapter 2, we discuss in more details the limitations of conventional QoS approaches in such an environment, and how our reward-based model addresses the problem.

In this thesis we propose a storage QoS performance model that freshly examines the issue of fair resource allocation in a heterogeneous environment in which server performance is highly dependent on workload characteristics (like SSD hit ratio). We believe that customers that move to a shared infrastructure will increasingly demand to see the benefits of better caching, smart data placement decisions, and good workload behavior, reflected in the performance of their applications in the shared environment, just as they would on a dedicated infrastructure. We adapt a QoS performance model called reward allocation that was proposed in $[12,13,14]$ as an approach to handle this situation. The idea behind our proposed reward-based QoS model is to explicitly favor applications that make more efficient use of the resources, rather than use the gains from one application to subsidize the performance of lesswell-performing applications. The subsidy model exemplified by proportional sharing is currently the most common solution for resource allocation in storage systems (see Chapter 2). However such solutions implicitly assume homogeneous resources and are less acceptable when server performance can fluctuate with application behavior. We explore the viability of our proposed scheduler in comparison to proportional scheduler when deployed with different scheduling models. In [12] we used sequential IO model, that does not adequately capture the concurrency available in a typical storage array or a clustered storage server. The work in [13] used the measured miss ratio to do reward scheduling in a model allowing concurrent device operations. The work in [14] uses the average latency to do reward scheduling in a model allowing 
concurrent device operations. Also we tried solving the problem of the difference in speed between read operations and write operations on SSD devices, by using our method.

The rest of this thesis is organized as follows. In Chapter 2 we motivate and define our problem and compare it with existing approaches. In Chapter 3 we introduce the main idea of the Reward Allocation Policy. In Chapter 4 we discuss our new allocation policy when used with storage servers only capable of sequential IO operations. In Chapter 5 we discuss our new allocation policy when used in a multi-tiered concurrent storage server model. In Chapter 6 we extend our allocation policy to handle client specific reservations and limits. We conclude in Chapter 7. 


\section{Chapter 2}

\section{Related Work}

There has been a significant research interest in developing scheduling algorithms and policies. Work in this area can be classified into four main areas: (i) Proportional share schedulers for networks and CPU; (ii) Proportional share schedulers for IO and disk scheduling; (iii) Time-Quanta based IO allocation; (iv) Multi-tag schemes.

\subsection{Proportional Share Schedulers For Networks and CPU}

In [15], Demers et al presented an analysis and simulation of their classical weighted fair queuing algorithm, and compared it to first-come-first-serve scheduling. In [16], a lottery-based scheduling algorithm for CPU resources was presented in this scheme, depending on the priority of an application it gets issued a number of lottery tickets. The resource is allocated to the client holding the winning ticket. In [17], the authors proposed a scheduling algorithm for integrated packet switching networks called starttime fair queuing. They proposed a computationally efficient scheduling algorithm that achieves fairness even with variable server's capacity.

\subsection{Proportional Share Schedulers For IO and Disk Schedul- ing}

The schemes presented in Section 2.1, have been extended to account for IO and disk scheduling. In [18], Cello; a disk scheduling framework was presented. Cello employ 
a two-level disk scheduling architecture; the first level consists of an applicationindependent scheduler, while the second level consists of a number of applicationdependent schedulers. In [19], a scheme to classify clients' requests into different performance categories, to provide different levels of performance guarantees was introduced. In [20],[21], the authors proposed Freeblock scheduling, which better utilizes the rotational latency periods and uses it to provide background media transfers, to achieve a higher disk bandwidth. In [22], a number of algorithms were introduced, with the target of giving a guarantee of a minimum share of the server's capacity with variable queue lengths. In [23], the authors introduced AVATAR, which is a low-level feedback-driven request scheduler fed by SARC, which is a high-level rate controller that isolates the clients' requests in a manner similar to [18]. In [24], the authors proposed pClock a scheduling algorithm that guarantees response times irrespective of the behavior of other clients. The scheme uses arrival curves to control the allowed bandwidth and burst characteristics of clients, and penalizes clients for misbehaving. In [25], the authors' proposed GSR, a real-time disk scheduling algorithm with the goal of optimizing the disk throughput while maintaining timing constraints. In [8], Gulati et al proposed PARDA, a proportional scheduler for distributed storage access.

\subsection{Time-Quanta Based IO Allocation}

In [26], the authors compared constant bandwidth allocation with proportional share resource allocation. In [27], CFQ the default scheduling algorithm for Red Hat Enterprise Linux 4 was proposed. CFQ distributes the bandwidth between processes using time-quanta based allocation. In [28], a quanta-based scheduling scheme to isolate slow SSD writes from faster reads was proposed, to enable a better utilization of SSD devices. In Fahrrad [29], a client quanta was defined, in terms of disk utilization to 
be reserved for each workload. The scheme in [30], used disk idling with tag synchronization to guarantee bandwidth and high throughput. In [31], Argon, a storage server the decrease the inefficiencies from inter-service disk and cache interference was proposed. Argaon guaranteed each client a fraction of the bandwidth they would get if they ran alone depending on the quantum. In [32], YFQ a scheduling algorithm that enables applications to ask for exclusive percentage of the disk bandwidth was proposed.

\subsection{Multi-Tag Schemes}

A multi-tag scheme for providing reservation and limit controls to proportional share schedulers were presented in [9]. Reservation and limit controls for storage servers were introduced in $[33,34,9]$. These models provide strict proportional allocation based on static shares (possibly subject to reservation and limit constraints). In [35] methods for accurate accounting of VM IO resource usage were presented.

These models provide strict proportional allocation based on static shares (possibly subject to reservation and limit constraints). In contrast, our work suggests changing shares to adapt to the behavior of the workload, rewarding well-behaved clients by targeted allocation rather than simply distributing the gains over all workloads. This characteristic is a desirable property of multi-tiered storage systems, where changes in access locality (and hit ratio) can drastically alter an application's profile in different execution phases. Time-quantum based approaches can be seen as a complementary method to our tag-based scheduling approach. The major issue with time quanta based allocation is the latency jitter caused by waiting for all remaining clients to finish their allocated quantum before scheduling pending requests. In contrast, the method in this thesis is a fine-grained allocation where client requests 
are interleaved at the level of individual requests, preventing the latency jitter. 


\section{Chapter 3}

\section{Reward Scheduling}

To motivate our idea, we start with a simple example. Consider two clients A and B, each having the same scheduling priority or weight. Each of them has a set of backlogged requests. For the example, we assume that the server consists of a single disk that can service a single request in $10 \mathrm{~ms}$. In any fair queuing scheduling algorithm, both clients will be awarded 50 IOPS (Input/Output Operations Per Second). If client $\mathrm{A}$ was able to have its requests serviced in $5 \mathrm{~ms}$ (perhaps using optimized patterns of IO access), a fair queuing algorithm would distribute the excess capacity generated by client A to both clients, effectively awarding each of them 66.7 IOPS.

At first glance, this may seem like a fair allocation. However, from client A's point of view, that is an unfair distribution of the excess capacity that if generated. Our proposed algorithm, Reward Scheduler, scales the weight of each client by an estimator that increases the weight of faster clients and decreases the weight of slower clients. For the scope of this example, lets use the average latency as the estimator for the Reward Scheduler. In our example, we only have a single disk that can service one request at a time, so the average latency would be equal to the the average service time. The Reward Scheduler will scale client A's weight by 5, while scaling client B's weight by 10 , effectively changing the weights from a $1: 1$ ratio to a $2: 1$ ratio, and thus awarding clients A and B 100 and 50 IOPS respectively. We can also see that by using Reward Scheduler the overall capacity of the system has been increased from 133.3 IOPS to 150 IOPS. 


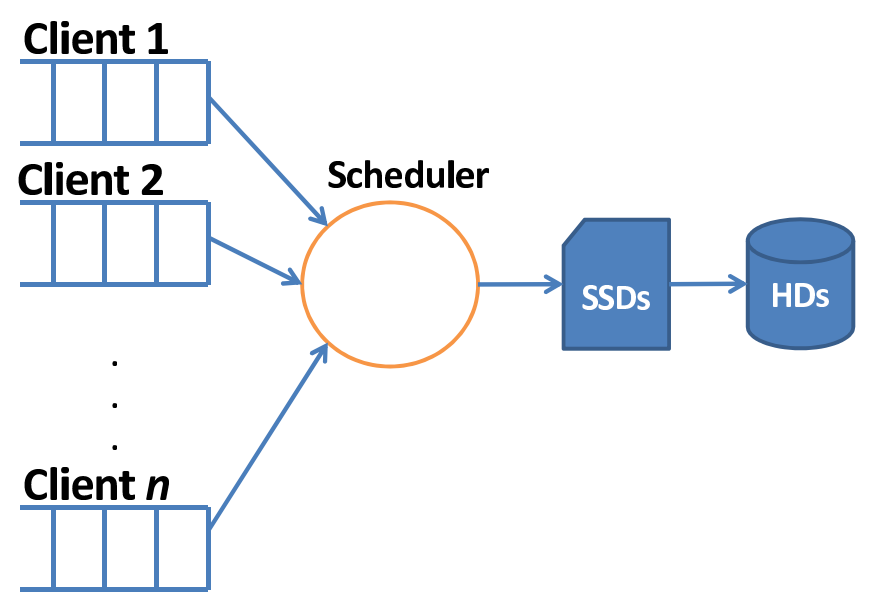

Figure 3.1 : Model for Sequential Storage

Different storage system models can be used with a Reward Scheduler. In [12], a sequential tiered storage system that can process a single request at a time was considered. A model of the system consisting of a front-end SSD cache and back-end disks is shown in Figure 3.1. If a request to the server is found in the SSD cache, it is termed a hit and served from the SSD; else it is a miss and is served from the hard disk subsystem. The average access times of the HD and SSD are $\Gamma$ and $\tau$ respectively.

A concurrent storage model was introduced in [13], as shown in Figure 3.2. Within the storage array the requests are sent to either the SSD or hard disk (HD) queue, from where they are served based on the array's scheduling priorities. The scheduler uses proportional share scheduling to choose which request to dispatch to the array next.

We will explore the ideas of tiered storage system capable of processing a single request at a time in Chapter 4 , and the usage of multi-tiered storage systems capable of concurrent IO operations in Chapter 5. 


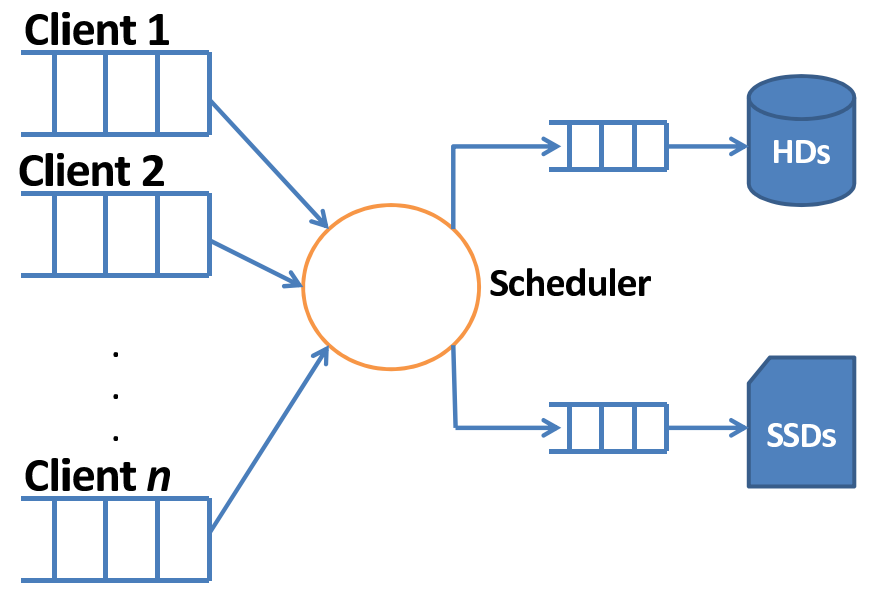

Figure 3.2 : Model for Multi-Tiered Storage 


\section{Chapter 4}

\section{Reward Allocation Policy in Sequential Model}

As mentioned before, the average access times of the HD and SSD are $\Gamma$ and $\tau$ respectively. There are $n$ clients that share the server, each client $j$ has a weight $\omega_{j}$. The weights represent the relative static priorities of the clients. The system maintains $n$ queues to hold pending requests, one for each client, as shown in Figure 3.1. Requests within a queue are served in FCFS order. When the server is free, the scheduler chooses the first request from one of the non-empty queues and dispatches it to the server. The server checks if the request is a SSD hit or a miss and serves the request from the appropriate device, in a sequential manner, one request at a time. We will present an experimental evaluation in Section 4.2

We now consider the performance of a single continuously-backlogged client running in isolation on this system. Define the hit ratio $h_{i}$ of client $i$ to be the fraction of its requests that are served from the SSD. The average service time for client $i$ is given by Equation 4.1. When run in isolation on the server, client $i$ will receive a throughput equal to $1 / \Phi_{i}$. We call this throughput the entitlement of client $i$, and denote it by $E_{i}$. Note that $E_{i}$ depends only on the client's hit ratio $h_{i}$ and speed of the devices.

$$
\Phi_{i}=\tau \times h_{i}+\Gamma \times\left(1-h_{i}\right) .
$$

When several clients are sharing the server, the aim of the scheduling policy is to 
try and provide clients with the same behavior that they would see in a dedicated system. If the system has sufficient capacity then each client should receive its entitlement. However, if the system has less than this capacity, our policy is to allocate the capacity to clients in proportion to their entitlements. The policy is formally specified below.

Reward Allocation Policy: Given a set of clients with static weights $\omega_{i}$ and entitlements $E_{i}$. The allocated capacity to the clients is in proportion to their weighted entitlements as $w_{i} \times E_{i}=w_{i} / \Phi_{i}, i=1, \cdots, n$. The allocations made by the Reward Algorithm Allocation Policy and Proportional Scheduling (PS) are summarized in Table 4.1. We denote by $\mathcal{A}$ the set of active clients, and consider an interval in which all active clients are continuously backlogged. We let $f_{i}$ denote the normalized weight of active client $i$. The value of $f_{i}$ is given by Equation 4.2. Note that the normalized weights must satisfy Equation 4.3.

$$
\begin{gathered}
f_{i}=w_{i} / \sum_{j \in \mathcal{A}} w_{j} . \\
\sum_{i \in \mathcal{A}} f_{i}=1 .
\end{gathered}
$$

It is easy to see why the throughputs stated in Table 4.1 hold. For reward scheduling, clients receive allocations in the ratio $r_{i}$ specified in Equation 4.4, and IOs of client $i$ require average time $\Phi_{i}$.

$$
r_{i}=\omega_{i} \times E_{i}
$$

Using Equation 4.4, we can deduce that the system performs a number of IOs $N_{R S}$ given by Equation 4.5, in a time given in Equation 4.6.

$$
N_{R S}=\sum_{i \in \mathcal{A}}\left(\omega_{i} \times E_{i}\right)
$$




\begin{tabular}{|c||c|c|}
\hline Policy & Total & Client $_{i}$ \\
\hline Reward Scheduler & $\sum_{i \in \mathcal{A}}\left(f_{i} \times E_{i}\right)$ & $E_{i} \times f_{i}$ \\
\hline PS & $1 / \sum_{i \in \mathcal{A}}\left(f_{i} / E_{i}\right)$ & $f_{i} / \sum_{i \in \mathcal{A}}\left(f_{i} / E_{i}\right)$ \\
\hline
\end{tabular}

Table 4.1 : Throughput Allocations under Reward Scheduler and PS.

$$
\sum_{i \in \mathcal{A}}\left(\omega_{i} \times E_{i} \times \Phi_{i}\right)=\sum_{i \in \mathcal{A}} \omega_{i}
$$

Now, using Equations 4.2 and 4.5, we can show that Equation 4.7 holds.

$$
\sum_{i \in \mathcal{A}}\left(\omega_{i} \times E_{i}\right)=\sum_{i \in \mathcal{A}}\left(f_{i} \times E_{i}\right) \times \sum_{j \in \mathcal{A}} \omega_{j}
$$

Hence, the total system throughput $T_{R S}$ is got by using Equation 4.6 and 4.7, and is equal to the quantity given in Equation 4.8

$$
T_{R S}=\sum_{i \in \mathcal{A}}\left(f_{i} \times E_{i}\right)
$$

For PS, the system performs $\sum_{i \in \mathcal{A}} \omega_{i}$ IOs in time given by Equation 4.9

$$
\sum_{i \in \mathcal{A}}\left(\omega_{i} \times \Phi_{i}\right)
$$

The total system capacity $T_{P S}$ is given in Equation 4.10

$$
T_{P S}=\sum_{i \in \mathcal{A}} \omega_{i} / \sum_{i \in \mathcal{A}}\left(\omega_{i} \times \Phi_{i}\right) .
$$

The capacity $C_{i}$ client $i$ equals to the quantity in Equation 4.11

$$
C_{i}=f_{i} \times \sum_{i \in \mathcal{A}} \omega_{i} / \sum_{i \in \mathcal{A}}\left(\omega_{i} \times \Phi_{i}\right)
$$


Using Equations 4.2 and 4.10 and simplifying Equation 4.11, we get client $i$ 's capacity given in Table 4.1 . Client $i$ 's capacity is equal to:

$$
C_{i}=f_{i} / \sum_{i \in \mathcal{A}}\left(f_{i} / E_{i}\right)
$$

Using Equation 4.3 and 4.12, the system throughput can be shown to be equal to the quantity given in Equation 4.13.

$$
T_{P S}=1 / \sum_{i \in \mathcal{A}}\left(f_{i} / E_{i}\right)
$$

\subsection{Reward Scheduling Algorithm}

The algorithm to implement Reward Scheduling is shown in Algorithm 1. Each client has a private queue to buffer its requests, until they are dispatched to the storage system. Each request is stamped with a tag which is used by the scheduler to arbitrate between the requests in different queues. Within a client's queue, the requests are served in FCFS order (arrival order). Hence, we only require to explicitly tag the request at the head of each client queue. We denote the tag of the first request of client $j$ as $\mathbf{s T a g}_{j}$. When invoked, the scheduler chooses the request with the minimum tag value to dispatch to the server.

The scheduler maintains statistics of the average latency of each client. We define latency as the time the request is dispatched from a client's queue to the time it finished service in the storage system. In this design, we track the average latency of the last $N$ (a configurable parameter) requests of each client. We denote the measured

average latency of client $j$ by $\bar{\phi}_{j}$. Clients with a low value of $\bar{\phi}_{j}$ get tags that are spaced closer together and are hence served more frequently than requests with larger latencies. 
Procedure RequestArrival shows what happens when a client $j$ sends a request $r$ at time $t$. If $j$ already has pending requests then $r$ is simply appended to the end of the queue, and its arrival time is noted. It will be assigned an sTag when the request reaches the head of the queue. On the other hand, if the request arrives to an empty queue, then it is assigned a tag equal to the larger of its arrival time and the sTag computed when the last request from that queue completed.

When a request (say from client $j$ ) completes service, the procedure RequestCompletion is invoked. The average latency of client $j, \bar{\Phi}_{j}$ is updated by incorporating the latency of the newly completed request. This is used to compute the next value of $\mathbf{s} \operatorname{Tag}_{j}$ by incrementing its current tag value by $\bar{\Phi}_{j} / \omega_{j}$. Thus the tags of successive requests of the client are spaced by an amount that is proportional to the average latency over the last $N$ requests, and inversely proportional to the static weight of the client. Hence clients who are completing their requests faster are given priority over those with slower requests, as are clients with higher static weights. The AdjustTags procedure is needed to handle the dynamic arrival and departure of clients. When a client joins the system by sending a request (either for the first time or after a period of inactivity), the tag assigned to this request needs to be synchronized with the tags of requests already in the system. Before a request is scheduled, the tags are adjusted so that the smallest tag is equal to the current real time, but the relative spacing of the tags is unchanged. That is, the tags are moved as a block to synchronize with the current time. This is the same synchronization mechanism used in $[9,24]$, and is used to prevent starvation of either the newly arriving request or the existing requests.

We consider a simple example of two Clients A and B with the same weights, and both using a single disk. Client B's requests are faster than those of client A. 


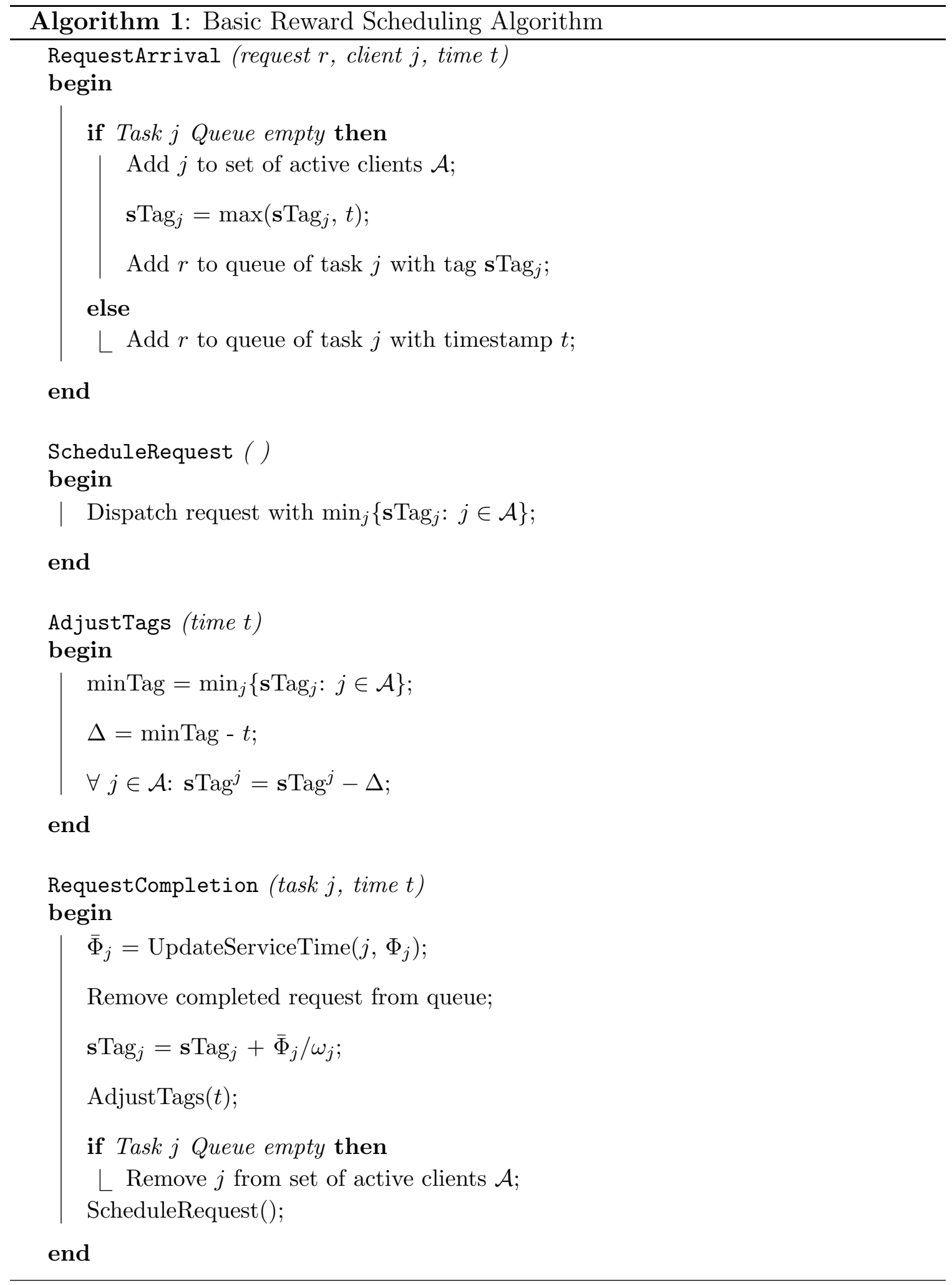




\begin{tabular}{|c|l|}
\hline Symbol & Meaning \\
\hline \hline $\mathcal{A}$ & Set of active tasks \\
\hline $\bar{\Phi}_{j}$ & Average latency of task $j$ \\
\hline$\omega_{j}$ & Static weight of Task $j$ \\
\hline sTag $_{j}$ & Scheduling tag of task $j$ \\
\hline
\end{tabular}

Table 4.2 : Symbols used by the Scheduler

time \begin{tabular}{|l|l|l|l|l|l|l}
0 & \multicolumn{1}{c}{10} & 15 & 25 & 30 & 40 & 45 \\
\hline A1 & B1 & & A2 & B2 & A3 & B3 $\ldots . .$. \\
\hline
\end{tabular}

Figure 4.1 : Requests Service Pattern using PS.

Assume client B's requests finish in $5 \mathrm{~ms}$ and client A's requests finish in $10 \mathrm{~ms}$. The service pattern using PS can be seen in Figure 4.1. At time $=0$, both clients have the same tag, the scheduler will choose one of them, assume it chooses A in case of a tie). After $10 \mathrm{~ms}, A_{1}$ finishes, and the tag of $A_{2}$ will be equal to the 2 . At time $10 \mathrm{~ms}$, the scheduler will dispatch $B_{1}$, which will finish at $t=15 \mathrm{~ms}$, and $B_{2}$ will be tagged to 2. At time $15 \mathrm{~ms}, A_{2}$ will be dispatched, and so on. It is clear that the PS scheduler allocated the capacity according to the weights only, and both clients will get 66.7 IOPS.

In contrast, if we used the reward scheduler with clients $\mathrm{A}$ and $\mathrm{B}$, we will get a new service pattern which can be seen in Figure 4.2. At time $=0$, both clients have the same tag, the scheduler will choose $A_{1}$. After $10 \mathrm{~ms}, A_{1}$ finishes, and its next tag will be equal to 20 . At time $10 \mathrm{~ms}$, the scheduler will dispatch B1, which will finish at $t=15 \mathrm{~ms}$, and the next tag will be 10 . The tags will get synchronized to current 


\begin{tabular}{ll|l|l|l|l|l|l|} 
time & \multicolumn{1}{c}{10} & 15 & 20 & 30 & 35 & 40 & 50 \\
\hline A1 & B1 & B2 & A2 & B3 & B4 & A3 \\
\hline
\end{tabular}

Figure 4.2 : Requests Service Pattern using Reward Scheduling.

time, so client A's tag will be 25 and client B's tag will be 15 . At time $15 \mathrm{~ms}, B_{2}$ will be dispatched as it has the lowest tag, and so on. It is clear that the reward scheduler allocated the capacity according to the service time, and client A got 50 IOPS, while client B got 100 IOPS.

\subsection{Experimental Evaluation}

In this section, we describe the empirical evaluation of our scheduling algorithm, Reward Scheduling, using a process-driven system simulator Yacsim [36]. Each client workload consists of a sequence of fixed-size block requests. The request service times are assumed to be uniformly distributed with a mean of $5 \mathrm{~ms}$ for a disk device, and a mean of $0.1 \mathrm{~ms}$ for the SSD. The workloads are all kept continuously backlogged with at least 1 outstanding request at any time. The experiments are designed to validate two aspects of the Reward Scheduling algorithm: (i) Guaranteeing weightbased allocation; (ii) Rewarding well behaved tasks

\subsubsection{Guaranteeing Weight Proportional Allocation}

This experiment demonstrates that Reward Scheduler reverts to a simple proportional scheduler when the clients have the same workload characteristics. Each client has a fixed static weight based on its SLA. At run time the Reward Scheduler generates the dynamic weight of the client by dividing it by the average service time of its 


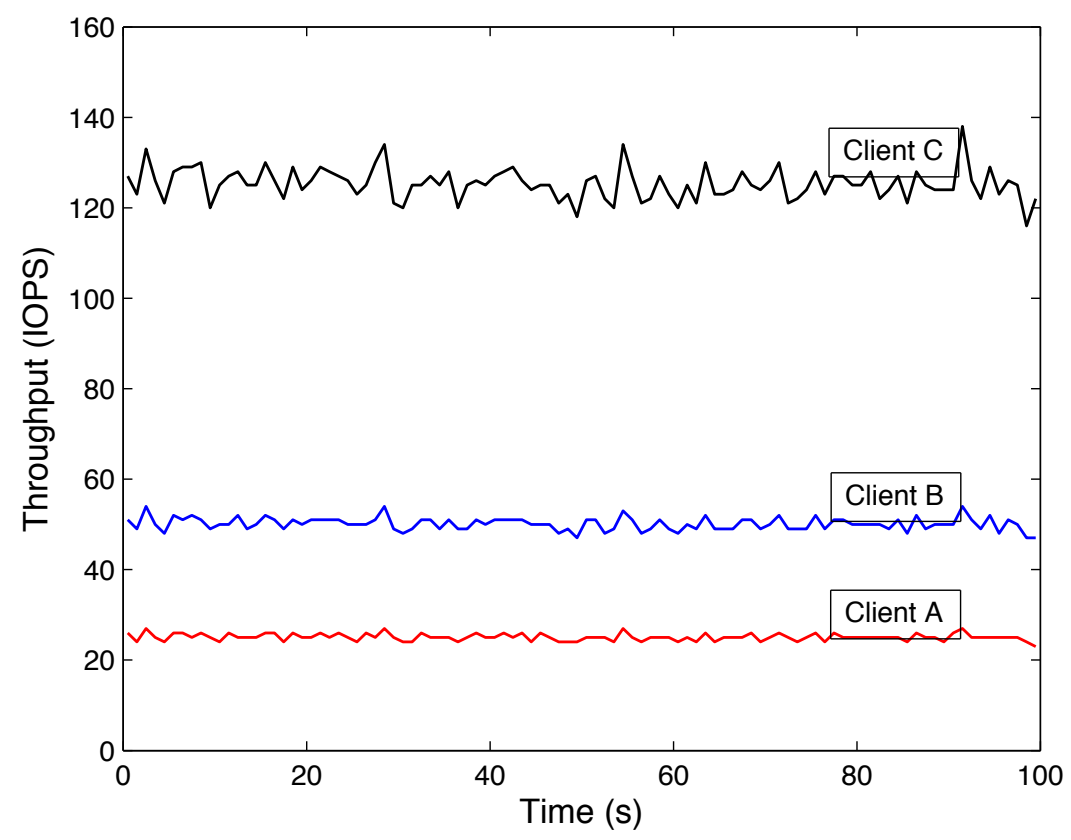

Figure 4.3 : Guaranteeing Weight Proportional Allocation

requests. When the clients have the same access profiles, the ratios of their dynamic weights and static weights are the same. We used three clients $\mathrm{A}, \mathrm{B}$ and $\mathrm{C}$ with static weights 1, 2 and 5 respectively. All the clients have a hit ratio of 0 (i.e. all disk requests) and reservations of 0. As shown in Figure 4.3, the Reward Scheduling algorithm successfully divides the capacity of the system for all clients in proportion to the weights. The capacity of the system is roughly 200 IOPS when the mean service time equals $5 \mathrm{~ms}$. The allocated bandwidths for the three clients are centered around 25, 50 and 125 IOPS respectively, in the ratio of their weights.

\subsubsection{Reward Scheduling}

Experiment 1: Rewarding workloads which have better runtime characteristics is a critical feature of the proposed algorithm. By reward we mean that the task with 
a shorter average service time is able to obtain a higher share of the IOPS of the server. As in the previous setup, the three clients A, B, and C have weights in the ratio $1: 2$ : 5, and are kept continuously backlogged. The hit ratios of $\mathrm{A}$ and $\mathrm{C}$ are kept constant at zero, as is the hit ratio of $\mathrm{B}$ before time $t=25$ and after time $t=75$. Between times $t=25$ and $t=75$, client B's hit ratio is at the higher value of 0.4. During this interval its average service time is equal to $3.04 \mathrm{~ms}$, compared to the average service time of $5.0 \mathrm{~ms}$ when all its requests are to the disk.

The throughput achieved by the tasks is shown in Figure 4.4(a). During the interval $[25,75]$ the bandwidth allocated to B increases from 50 IOPS to roughly 83 IOPS, while the throughputs of clients A and C remain constant at 25 and 125 IOPS respectively throughout the experiment. The increase in B's allocation is directly related to the decrease in its service time by a factor of $3 / 5$ that increases its throughput by a factor $5 / 3$, while leaving the other allocations unchanged. This is the main feature in our algorithm. In Figure 4.4(b) we show the same scenario but using a Proportional Scheduler. During the interval $[25,75]$, the system capacity increases a little from 150 to 222 IOPS. This increase is proportionally distributed among the three clients, which all show a small increase in their throughput. Meanwhile client B which was the main cause for this increase in the total bandwidth, was barely rewarded, as shown by comparing its allocation during this interval in Figures 4.4(a) and 4.4(b).

Experiment 2: Consider two clients $\mathrm{A}$ and $\mathrm{B}$ of equal weight that are run on a server with characteristics $\Gamma=5 \mathrm{~ms}$ and $\tau=0.1 \mathrm{~ms}$. Each client is kept continuously backlogged.

The time interval is divided into four regions, and a client's hit ratio is different in each region. Client $\mathrm{A}$ has a hit ratio of $0,1 / 5,2 / 5$ and $3 / 5$ in regions 1 through 4 respectively, while client $\mathrm{B}$ has hit ratios of $2 / 3,1 / 2,1 / 3$ and 0 . Figure 4.5 shows the 


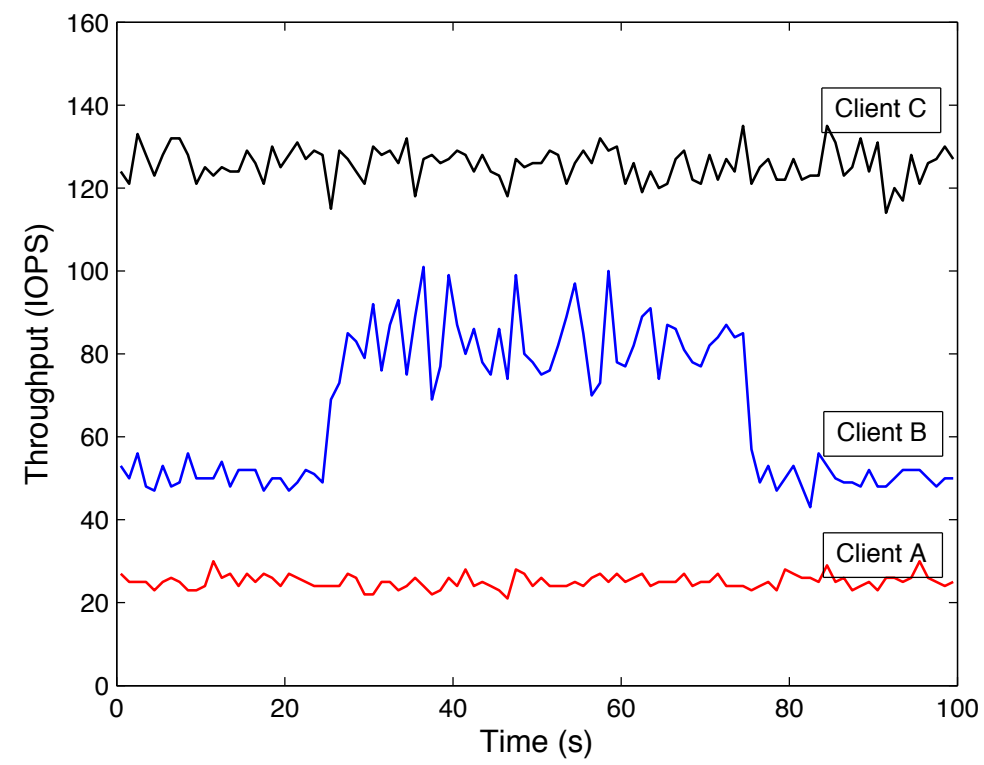

(a) Allocation by Reward Scheduler

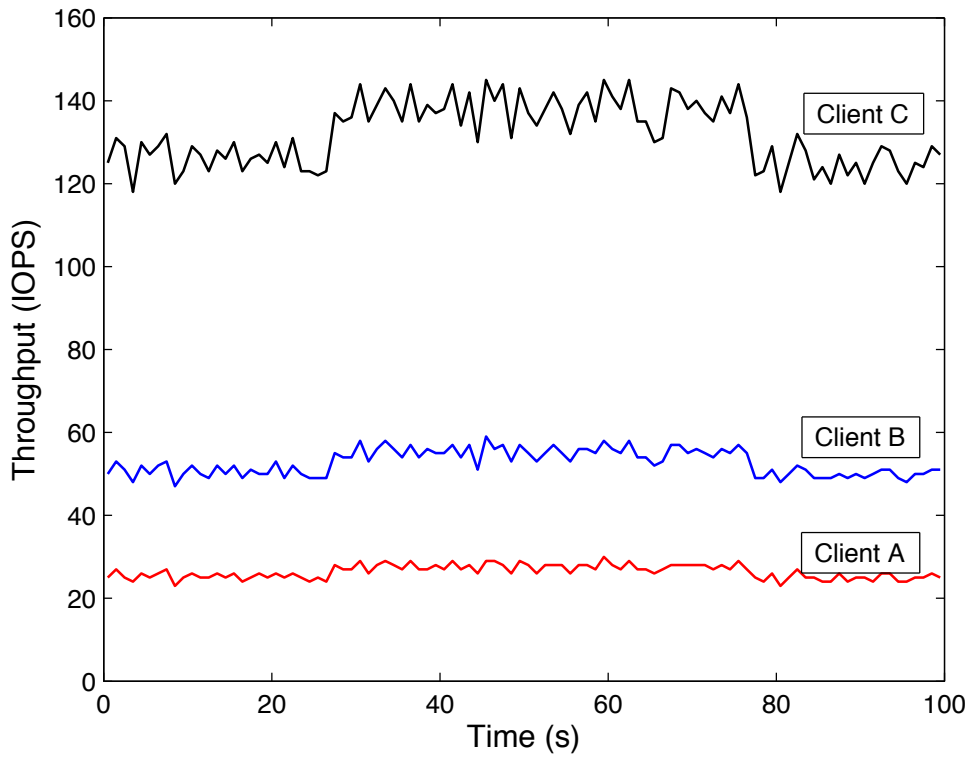

(b) Allocation by Fair Scheduler

Figure 4.4 : Comparison of Capacity Allocation in PS and Reward Schedulers 


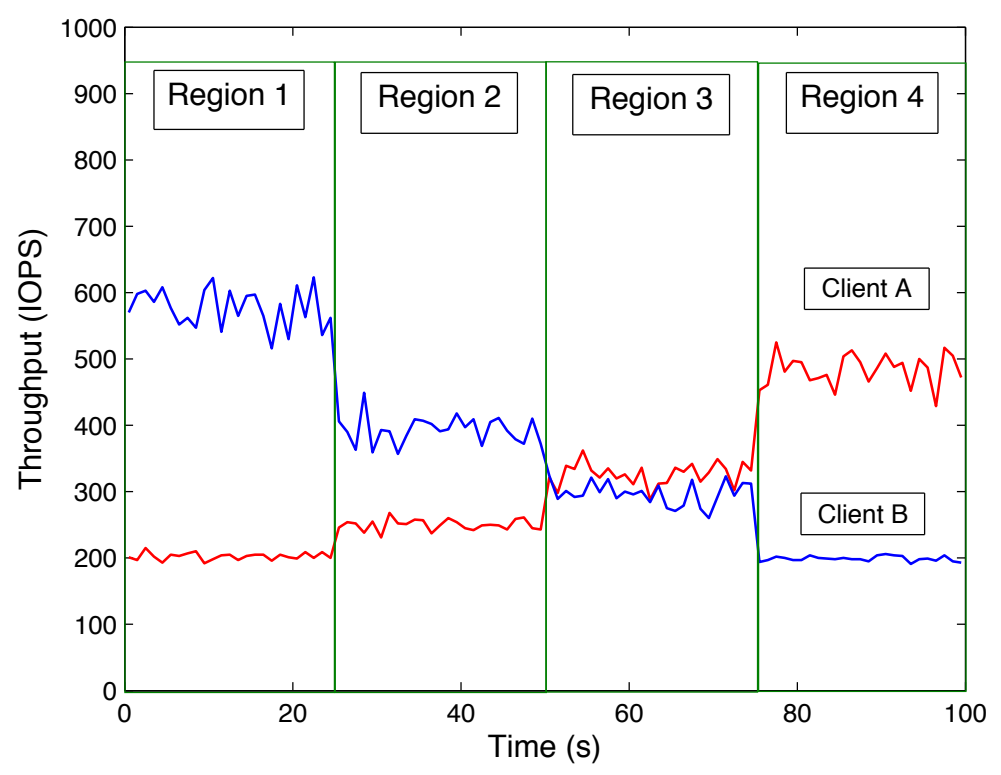

Figure 4.5: A and B run separately. Throughput is the clients entitlement.

throughput achieved by $\mathrm{A}$ and $\mathrm{B}$ in each region when run separately by itself on the system. By definition this is the entitlement of the client, and it depends only on its hit ratio and the device speeds. Figures 4.6(a) and 4.6(b) show the throughputs of A and B when both clients are run together on the same server, using PS and RAP respectively. Under PS the clients receives equal bandwidth because their weights are the same and the relative allocations made by PS are insensitive to hit ratio. The differing hit ratios change the overall system capacity a little as shown in the legend in each region. In contrast, RAP behaves very differently, and in each region gives A and $\mathrm{B}$ service in proportion to their entitlements in that region. Hence, the form of Figure 4.5 and Figure 4.6(b) are identical except that each throughput is divided by two since two equal-weight clients are sharing the server. The total system throughput in each region also exceeds the system throughput under PS as expected. One can 


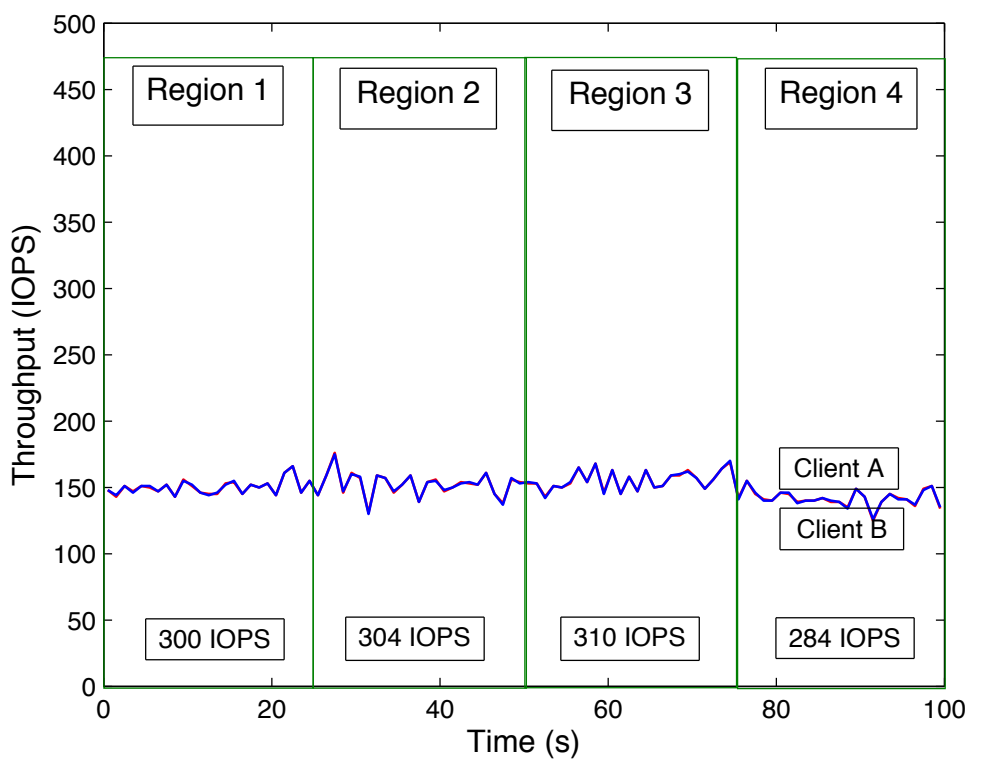

(a) Clients A and B run together using PS.

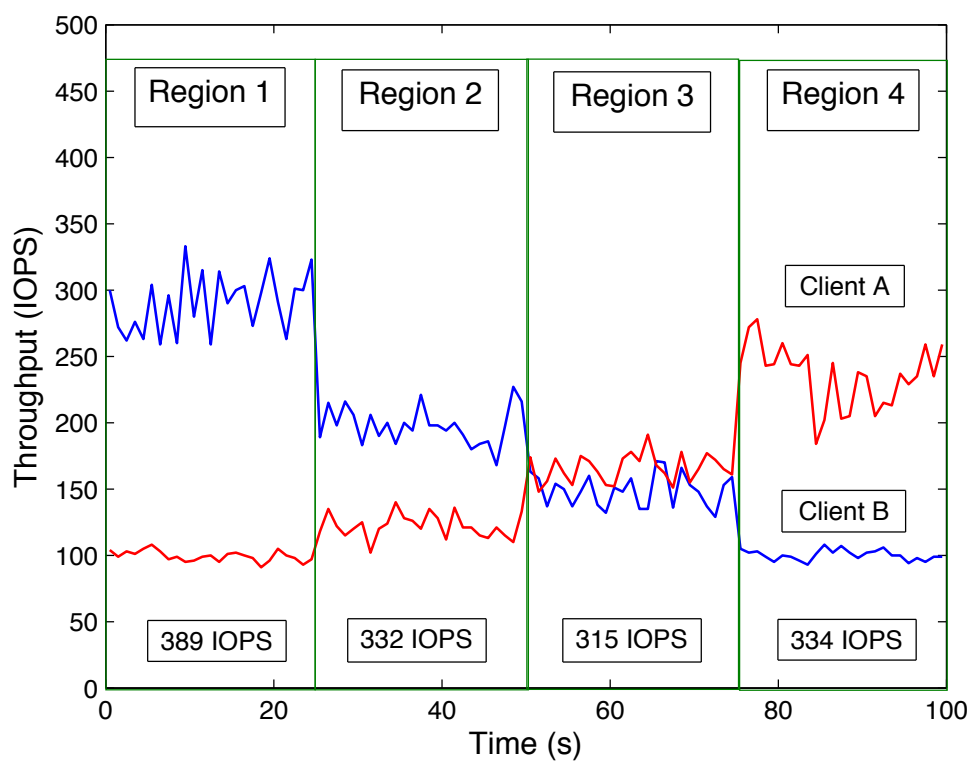

(b) Clients A and B run together using RAP.

Figure 4.6 : Comparison of allocation when clients are run separately using PS and RAP. PS does not distinguish the behavior of the clients within a region, while the allocation by RAS is proportional to the clients' entitlements. Overall system throughput under RAP exceeds that under PS. 


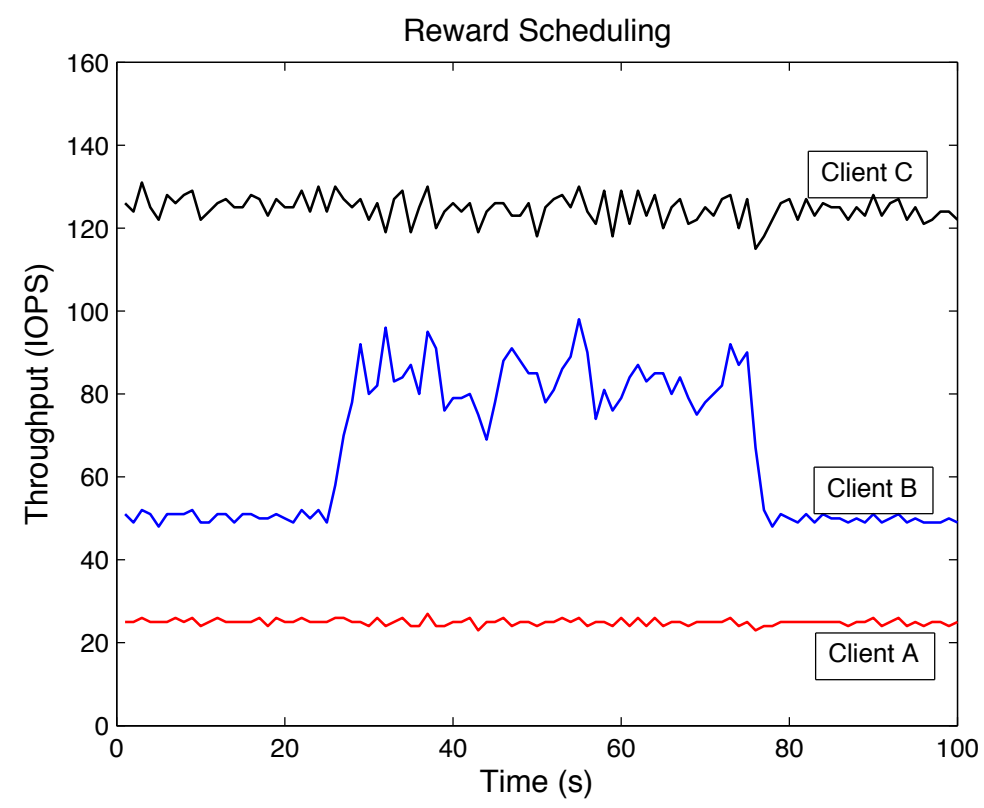

Figure 4.7 : Reward Scheduling with Hit Ratio.

verify that the results are consistent with the entries in Table 4.1.

Experiment 3: Using the same parameters as in Experiment 1, we use the hit ratio as the main performance parameter for each client. This experiment shows that the hit ratio could be used as a viable performance parameter.

\subsection{Summary}

In this chapter, we proved analytically the viability of the reward allocation policy to be used with tiered sequential storage architectures. Mainly by using average latency as the main performance parameter, but also by using hit ratio as one of the viable parameters to be used in our reward scheduler. 


\section{Chapter 5}

\section{Reward Allocation Policy in Concurrent Model}

As mentioned before the average access times of the HD and SSD are $\Gamma$ and $\tau$ respectively. There are $n$ clients that share the server. Each client $j$ has a weight $\omega_{j}$. The weights represent the relative static priorities of the clients. The system maintains $n$ queues to hold pending requests, one for each client, as shown in Figure 3.2. Requests within a queue are served in FCFS order. When the server is free, the scheduler chooses the first request from one of the non-empty queues and dispatches it to the server. The server checks if the request is a SSD hit or a miss and serves the request from the appropriate device. Ideally all storage devices will be fully utilized. We will present an experimental evaluation in Section 5.2, which will include experiments using average latency as the main performance parameter. We define the ratio between the HD average access time to the SSD average access time as $\alpha$ as shown in Equation 5.1. Note that $\alpha$ is governed by the the Inequality 5.2. The hit ratio of client $i^{\prime} s$ workload $\left(h_{i}\right)$ is the fraction of its IO requests that are hits. The miss ratio is defined by Equation 5.3.

$$
\begin{gathered}
\alpha=\Gamma / \tau . \\
\alpha>1 . \\
m_{i}=1-h_{i} .
\end{gathered}
$$


We first consider the performance of a single continuously-backlogged client running in isolation, as a function of its hit ratio, on a multi-tiered storage server, where requests can be serviced in parallel on the SSDs and HDs. The throughput increases as the hit ratio improves until a threshold value for the hit ratio $\alpha /(\alpha+1)$ is reached, after which it decreases again. As defined before, $\alpha$ is the speed difference ratio in the storage server. In the first region where hit ratio $<\alpha /(\alpha+1)$ the $\mathrm{SSD}$ is not fully utilized, as the number of requests intended for the SSD are serviced in less time than those intended for the HD. The behavior is confirmed by simulation results in Figure 5.1. The service times are exponentially distributed random variables with mean values $\Gamma=10 \mathrm{~ms}$ and $\tau=5 \mathrm{~ms}$ respectively. Requests of client $i$ are independently labeled hits (misses) with probability $h_{i}\left(m_{i}\right)$. The turning point at the threshold occurs when the load on the SSD is high enough to overwhelm its higher speed. At the threshold hit ratio, the total service times for all the requests on each device are equal; beyond that, the SSD becomes the bottleneck device. In this example $\alpha$ is equal to $2 / 3$. Figure 5.2 , shows the throughput curve when $\Gamma=10 \mathrm{~ms}$ and $\tau=5 \mathrm{~ms}$, and $\alpha=5 / 6$. Typical values of these parameters for read accesses are $0.1 \mathrm{~ms}$ and $10 \mathrm{~ms}$ respectively. In this case $\alpha$ will be equal to 0.99 . Figure 5.3, shows the throughput allocation with different hit ratios. We will focus on the range $[0, \alpha / \alpha+1]$ for the range of hit ratios used in our proofs and experiments.

Reward Allocation Policy: We now discuss the case when several clients with different hit ratios are sharing the storage system. Recall that the aim of the scheduling policy is to provide clients with the same behavior as they would in a dedicated system. If the system had enough capacity then each client should receive its entitlement. However, if the system has less than this capacity, our policy, enforces a strong isolation policy, that is to allocate the capacity to clients in proportion to 


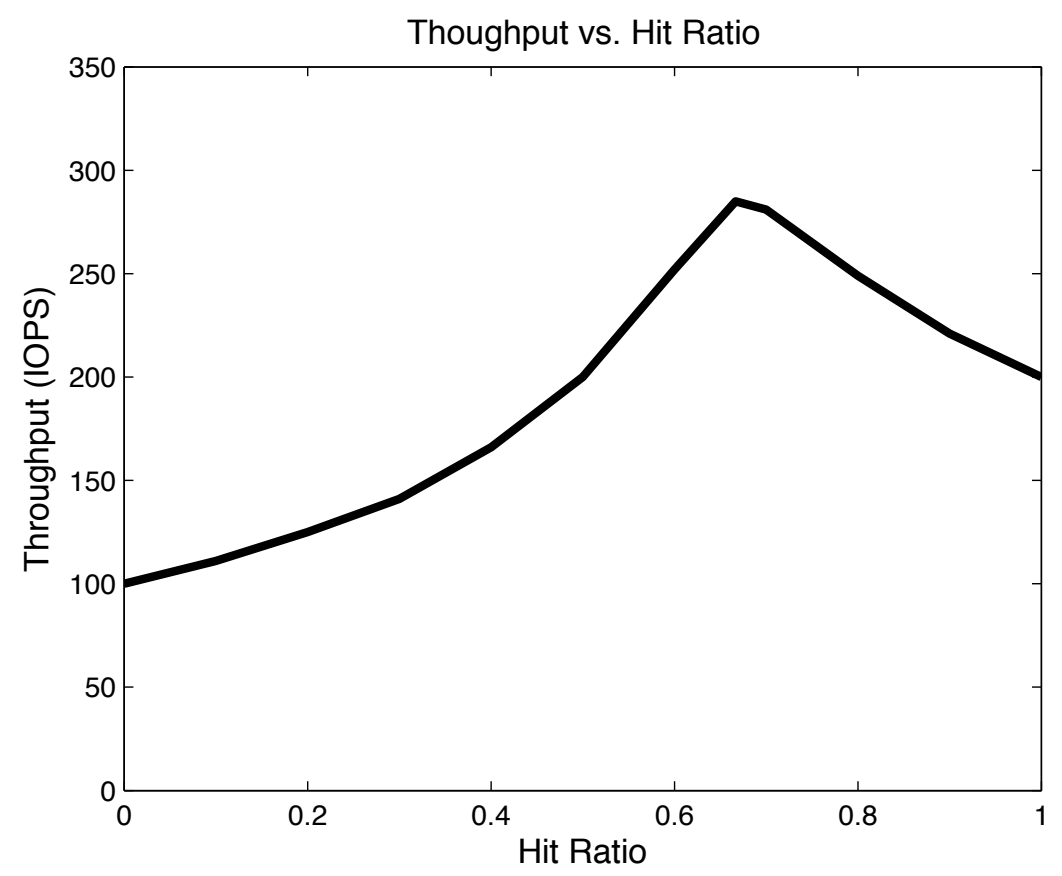

Figure 5.1 : Throughput vs. Hit ratio, $\alpha=2 / 3$

their entitlements. For example, consider two clients with $h_{i}=1 / 4$ and $h_{j}=2 / 3$ and storage parameters of Figure 5.1. The entitlements of $i$ and $j$ are 133.3 and 300 IOPS respectively. Hence, Reward Scheduling will allocate throughput in the ratio 133.3 : 300 (i.e. $4: 9$ ).

The following are the properties of reward scheduling based allocation, on a mutlitiered storage server. The set of clients in $\mathcal{A}$ are continuously backlogged with hits distributed at fine granularity in proportion to $h_{i} \in[0, \alpha / \alpha+1]$, with equal arbitrary weights $w_{i}$. Property 3 clearly shows the isolating behavior of Reward Scheduling. The absolute throughput of client $i$ depends only on its own workload (miss ratio and the device speeds). It is not affected by the changes in other workloads, but changes in tandem with its own workloads characteristics. 


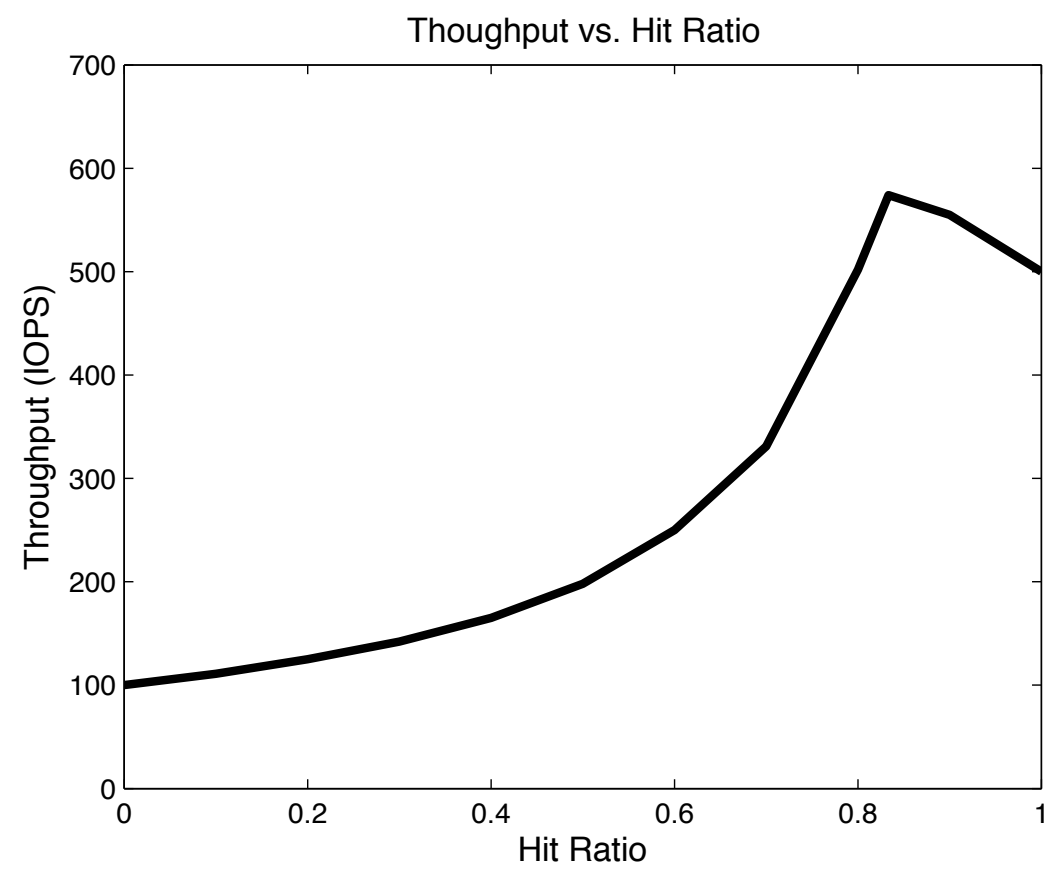

Figure 5.2 : Throughput vs. Hit ratio, $\alpha=5 / 6$

1. The entitlement of client $i$ (throughput when running in isolation) is $\frac{1}{m_{i} \times \Gamma}$.

2. The throughput of client $i$ is given by $\frac{f_{i}}{m_{i} \times \Gamma}$.

3. The total system throughput is given by $\frac{1}{\Gamma} \sum_{i \in \mathcal{A}} \frac{f_{i}}{m_{i}}$.

To prove property number 1 , and knowing that we are operating in the region $h_{i} \in[0, \alpha / \alpha+1]$, we know than the bottle neck device is the HD, so in time equal to $\Gamma$ the number of request serviced $k_{i}$ equal to Equation 5.4.

$$
k_{i}=1+\left(h_{i} / m_{i}\right) .
$$

Simplifying Equation 5.4 gives us Equation 5.5

$$
k_{i}=\left(m_{i} / m_{i}\right)+\left(h_{i} / m_{i}\right)=1 / m_{i} .
$$




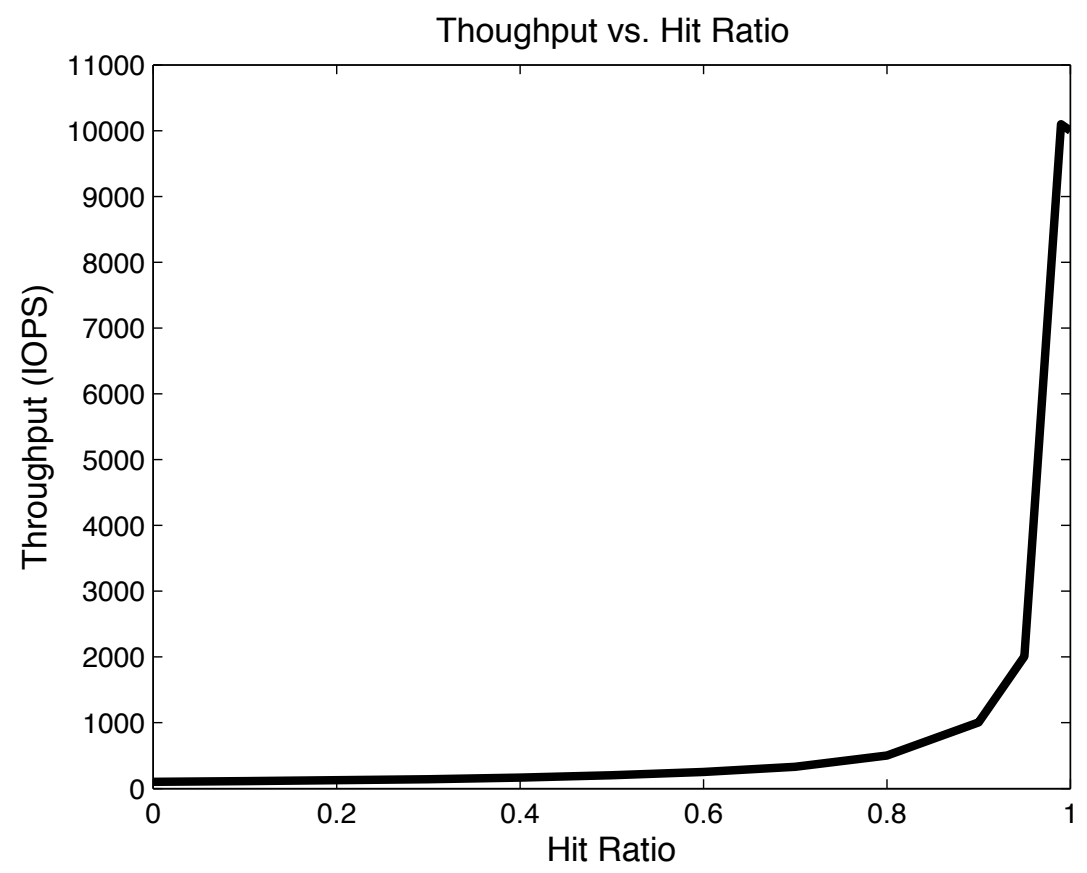

Figure 5.3 : Throughput vs. Hit ratio, $\alpha=0.99$

Also as the HD is the bottle neck device it is clear that Equation 5.6 holds.

$$
\Gamma \times m_{i}>\tau \times h_{i}
$$

Using Equations 5.5 and 5.6 we can calculate that the entitlement $E_{i}$ of client $i$ is equal to Equation 5.7, which is property 1.

$$
E_{i}=\frac{1}{m_{i} \times \Gamma}
$$

To prove property 2, we recall that the normalized weight of each client is $f_{i}$. Using the knowledge that the reward scheduler allocates capacity according to the ratio of weighted entitlements. Client $i$ 's capacity $C_{i}$ requests in time $T_{R S} . C_{i}$ is equal to Equation 5.8, and $T_{R S}$ is given in Equation 5.9

$$
C_{i}=f_{i} \times E_{i}
$$




$$
T_{R S}=\sum_{i \in \mathcal{A}} f_{i} \times m_{i} \times E_{i} \times \Gamma
$$

Simplifying Equation 5.9, we get that $T_{R S}=1$. Using Equations 5.8 and 5.7 we can prove that client $i$ 's bandwidth allocation in a shared environment is equal to:

$$
C_{i}=\frac{f_{i}}{m_{i} \times \Gamma}
$$

By simply using the summation of all clients' throughput from Equation 5.10, we can prove that the total system throughput is equal to:

$$
K_{i}=\frac{1}{\Gamma} \sum_{i \in \mathcal{A}} \frac{f_{i}}{m_{i}}
$$

\subsection{Reward Scheduling Algorithm}

The reward scheduling algorithm to implement Reward Scheduling using the average miss ratio is shown in Algorithm 2. All functions are the same as explained in Section 4.1, except that the scheduler maintains statistics of the average hit ratio of each client. In this design we track the average hit ratio of the last $N$ (a configurable parameter) requests of each client. We denote the measured average hit ratio of client $j$ by $h_{j}$. Clients with a low value of $h_{j}$ get tags that are spaced further apart and are hence served less frequently than requests with larger hit ratios.

When a request (say from client $j$ ) completes service, the procedure RequestCompletion is invoked. The average hit ratio of client $j, h_{j}$ is updated by incorporating the information about the device that serviced the newly completed request. This is used to compute the next value of $\mathbf{s} \operatorname{Tag}_{j}$ by incrementing its current tag value by $\left(1-h_{j}\right) \times \Gamma /\left(\omega_{j}\right)$. Thus the tags of successive requests of the client are spaced by an amount that is proportional to the average miss ratio over the last $N$ 


\begin{tabular}{|c|l|}
\hline Symbol & Meaning \\
\hline \hline $\mathcal{A}$ & Set of active tasks \\
\hline$h_{i}$ & Average hit ratio of task $j$ \\
\hline$\omega_{j}$ & Static weight of Task $j$ \\
\hline sTag $_{j}$ & Scheduling tag of task $j$ \\
\hline
\end{tabular}

Table 5.1 : Symbols used by the Scheduler

requests, and inversely proportional to the static weight of the client, and the HD average access time. Hence clients who are having their requests serviced from the faster SSD are given priority over those with a lower hit ratio requests, as are clients with higher static weights.

A simple example of two Clients A and B, both using a single disk and a single SSD, which can service request in parallel. Both clients have the equal weights equal to 0.5 , but client B's hit ratio is equal to 0.5 , and client A's hit ratio is equal to 0 . Service times for HD and SSD are $\Gamma=10 \mathrm{~ms}$ and $\tau=5 \mathrm{~ms}$ respectively. The service pattern using PS can be seen in Figure 5.4. At time $=0$, both clients have the same tag, the scheduler will choose $A_{1}$. After $10 \mathrm{~ms}, A_{1}$ finishes, and the tag will be equal to 2. At time $10 \mathrm{~ms}$, the scheduler will dispatch $B_{1}$ to the $\mathrm{HD}$ and schedule $A_{2}$ to be dispatched next. $B_{1}$ will finish at $t=20 \mathrm{~ms}$, and $B_{2}$ tagged to 2 . At time $20 \mathrm{~ms}, A_{2}$ will be dispatched to the $\mathrm{HD}$ and $B_{2}$ to the $\mathrm{SSD}$, and so on. It is clear that the PS scheduler allocated the capacity according to the weights only, and both clients will get 66.7 IOPS.

In contrast, if used the reward scheduler with clients $\mathrm{A}$ and $\mathrm{B}$, we will get a new service pattern which can be seen in Figure 5.5. At time 0, both clients have the same 


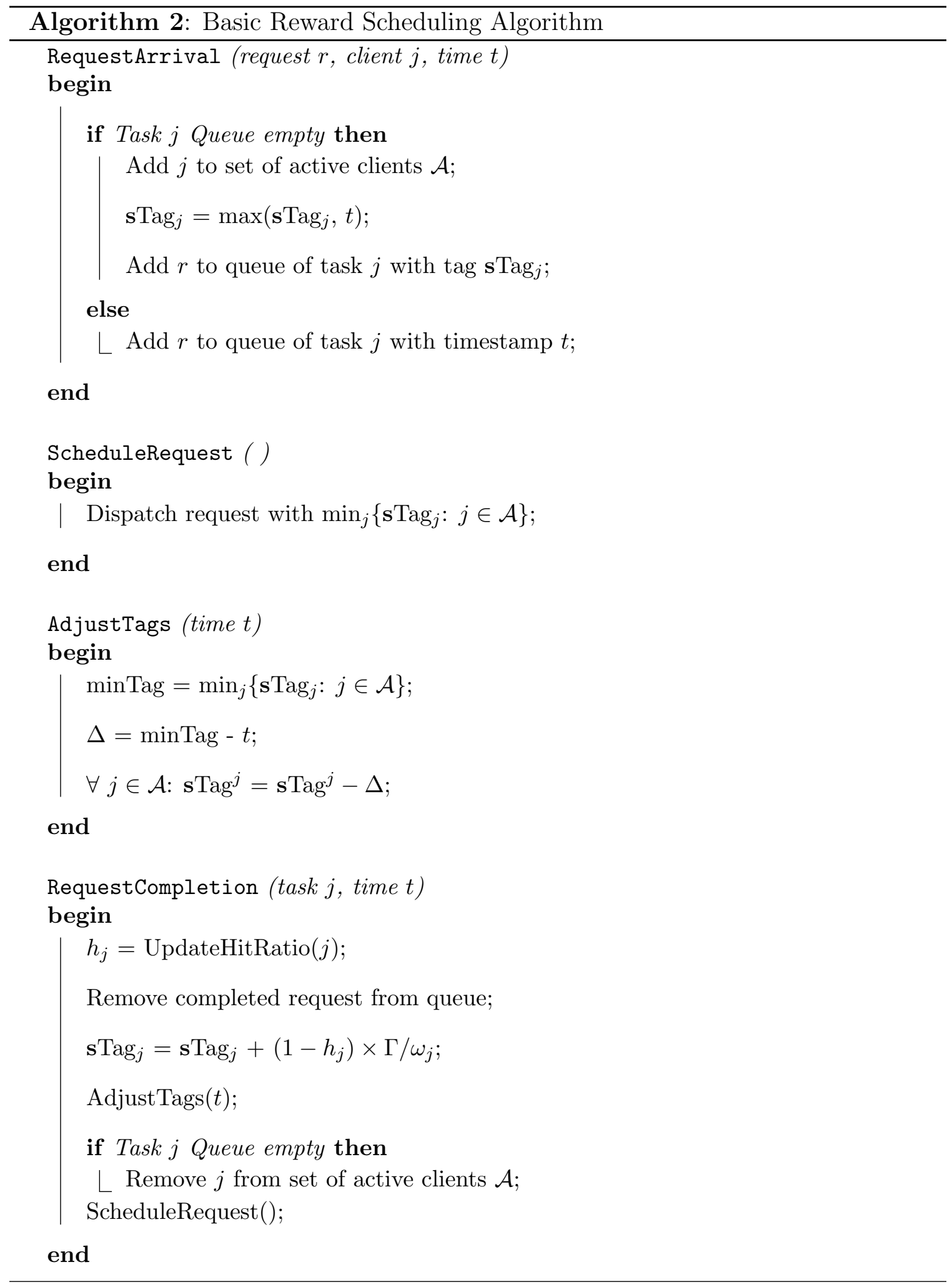




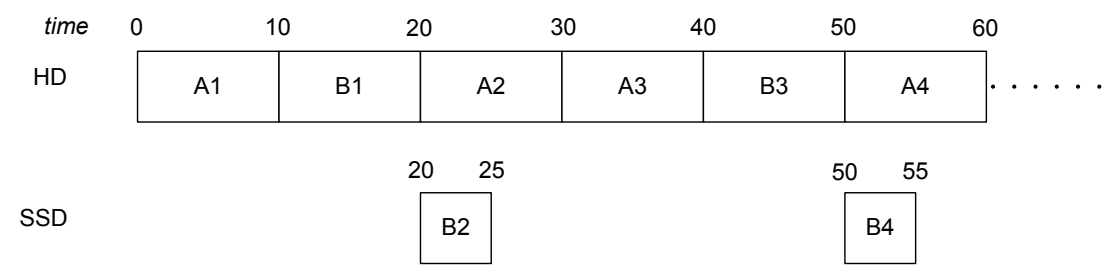

Figure 5.4 : Requests Service Pattern using PS.

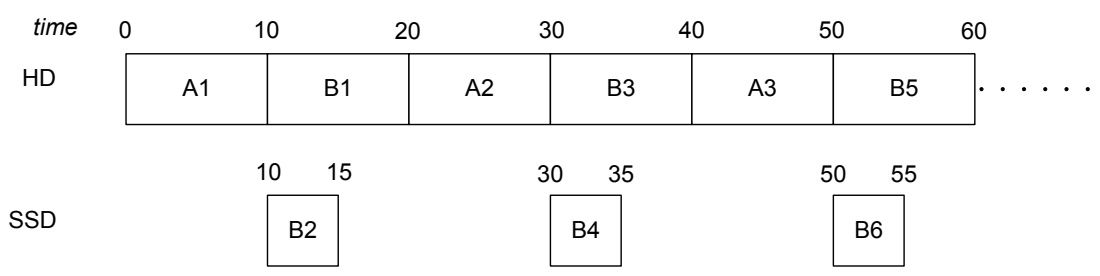

Figure 5.5 : Requests Service Pattern using Reward Scheduling.

tag, the scheduler will choose $A_{1}$. After $10 \mathrm{~ms}, A_{1}$ finishes, and the tag of $A_{2}$ will be equal to the 0.2 . At time $10 \mathrm{~ms}$, the scheduler will dispatch $B_{1}$ to the $\mathrm{HD}$, and $B_{2}$ to the SSD because of the tags. B2 will finish at $t=15 \mathrm{~ms}$, and $B_{3}$ will be tagged to 0.1 , after that the tags are synchronized to the current time. At time $15 \mathrm{~ms}, A_{2}$ will be scheduled to run next as it has the lowest tag, and so on. It is clear that the reward scheduler allocates the capacity according to the hit ratio, and client $\mathrm{A}$ got 50 IOPS, while client B got 100 IOPS.

\subsection{Experimental Evaluation}

We performed simulation experiments to show the behavior of the system with exponential service times and uniformly distributed cache hits. The scheduler monitors the hit ratio of each workload over a moving window of 100 requests. Requests of client $i$ are labeled with tags that are spaced by $m_{i}$, where $m_{i}$ is the current measured 
miss ratio for that client's workload. The requests are serviced in increasing order of their tag values.

Experiment 1: Figure 5.6(a) and Figure 5.6(b) show the results for proportional share and Reward based scheduling algorithms respectively. The system contains a single disk and a single SSD, with $\Gamma=10 \mathrm{~ms}$ and $\tau=5 \mathrm{~ms}$. The clients have a hit ratio of 0 except during the time interval 100 to 200 seconds when A's workload has a hit ratio of 0.5 . The static weights of the workload are in the ratio $1: 1$. With proportional scheduling, both A and B increased their throughput to roughly 67 IOPS during 100 - 200s interval. However under Reward Scheduling, B is not affected by the changes in A's hit ratio, and it maintains a constant 50 IOPS. Client A has increased its throughput from 50 to to roughly 100 IOPS as predicted. The results for device utilizations (Figure 5.7) match the analytic framework we discussed earlier.

Experiment 2: We use the same system as in Experiment 1, but with the static weights of the workloads in the ratio $2: 1$. Moreover client A's hit ratio increased to 0.6 in the 100 - 200s interval. As shown in Figure 5.8, the scheduler in the time range 100 to 200 correctly distributed the capacity according to the weights and hit ratios, and award client B 90 IOPS. By using Equation 5.10, we can calculate the capacity of B to 83.3 IOPS. Meanwhile Client A's capacity didn't change. Experiment 3: In this experiment we used a SSD as the storage device and we vary the read write (RW) ratio. Both workloads have equal weights and RW ratio equal to 0.5. At time 5s, workload 1's RW ratio changes to 1.0. Figure 5.9(a) shows the behavior of the two workloads when the disk queue was set to 1 . The reward behavior successfully rewarded workload 1 with the extra capacity. We then changed the disk queue to see the behavior. Figure 5.9(b) shows the allocation when the disk queue was set to 8 . We see that workload 2 get some of the gains and the gain of 1 reduced compared 


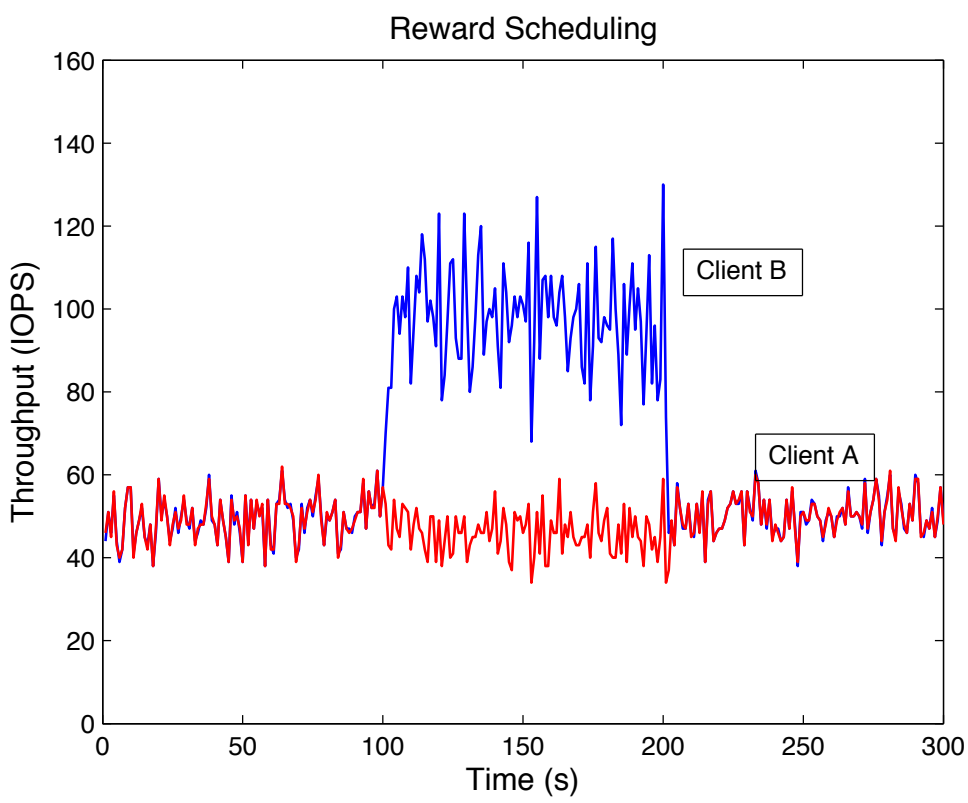

(a) Reward Scheduler.

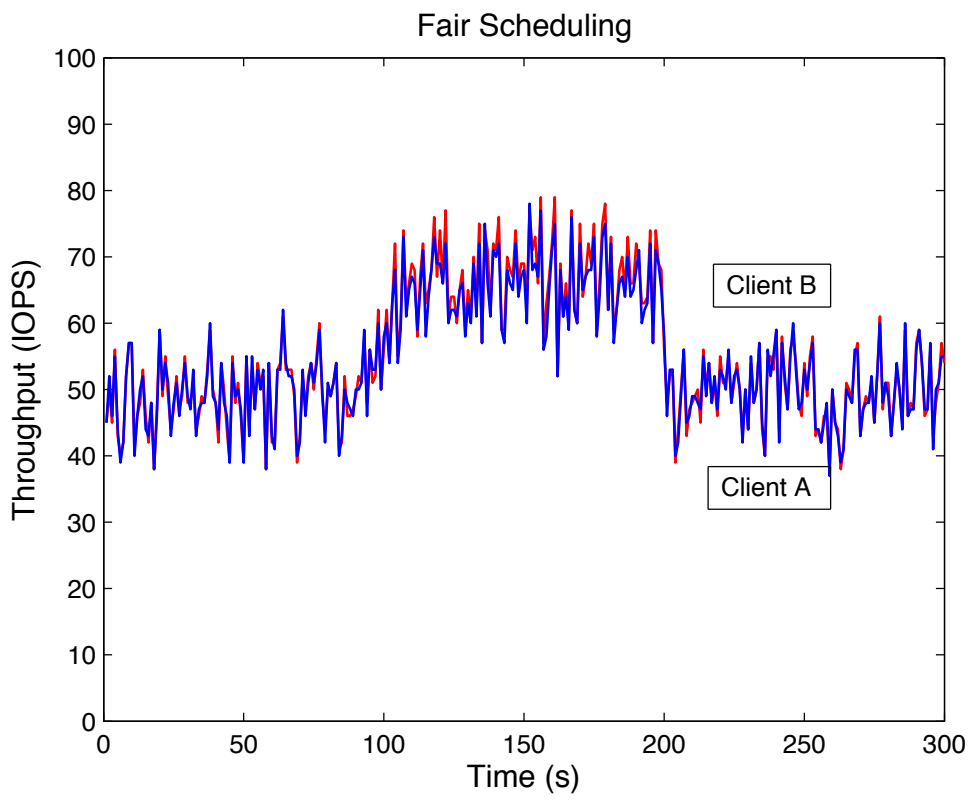

(b) Proportional Scheduler.

Figure 5.6 : Comparison of allocation while using Hit Ratio as a scaling parameter. 


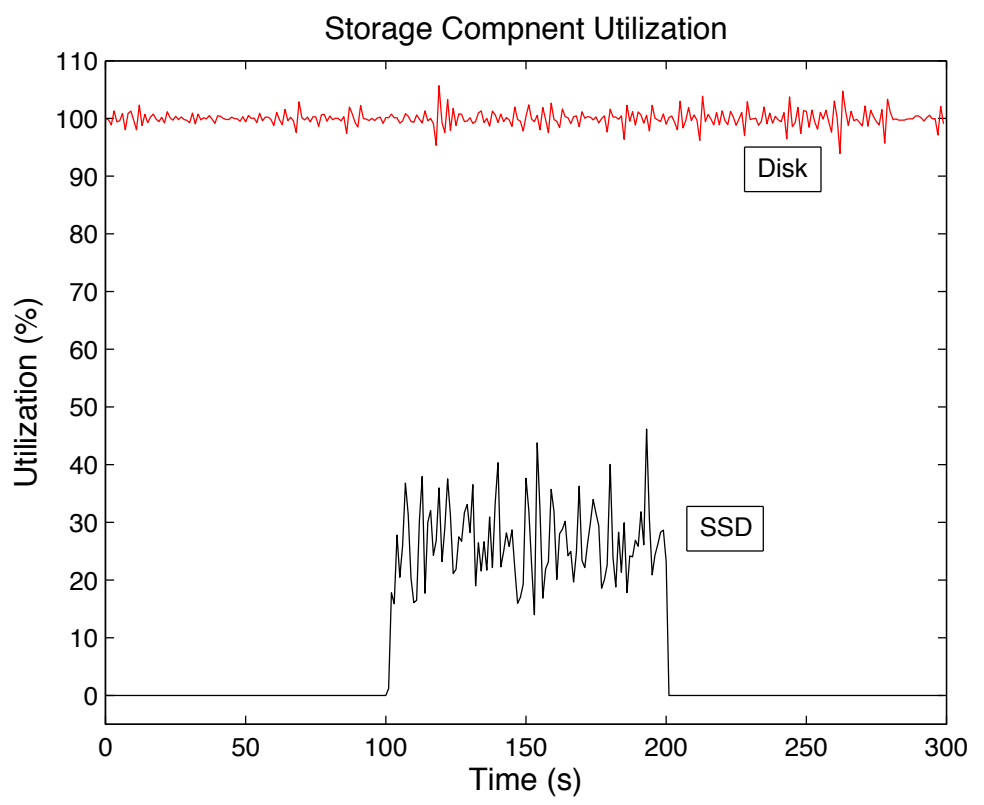

Figure 5.7 : Device Utilizations.

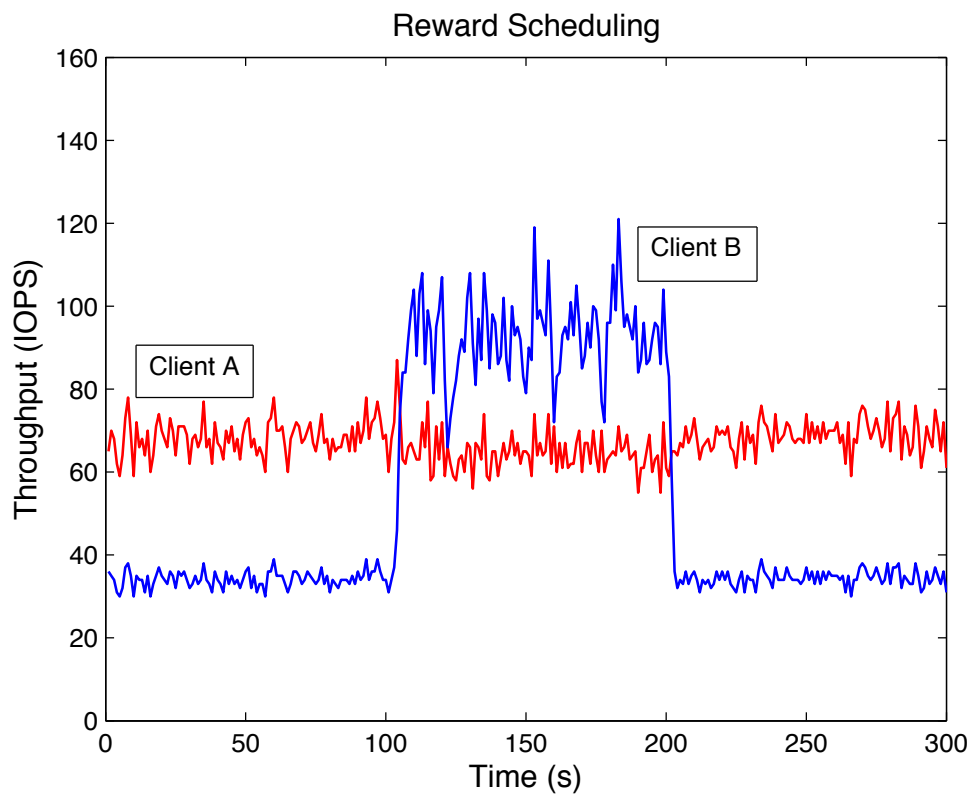

Figure 5.8: Reward Scheduling with Arbitrary Weights. 
to (a). The reason is because the scaling factor gets diluted as the queue length gets larger, since all of them include the fixed portion of the delay introduced by queuing the requests in the storage queue.

\subsection{Summary}

In this chapter, we proved analytically the viability of the reward allocation policy to be used with multi-tiered concurrent storage architectures. Mainly by using hit ratio as the main performance parameter, we showed that allocations proportional to entitlements can be achieved for workloads with the same dominant device. 


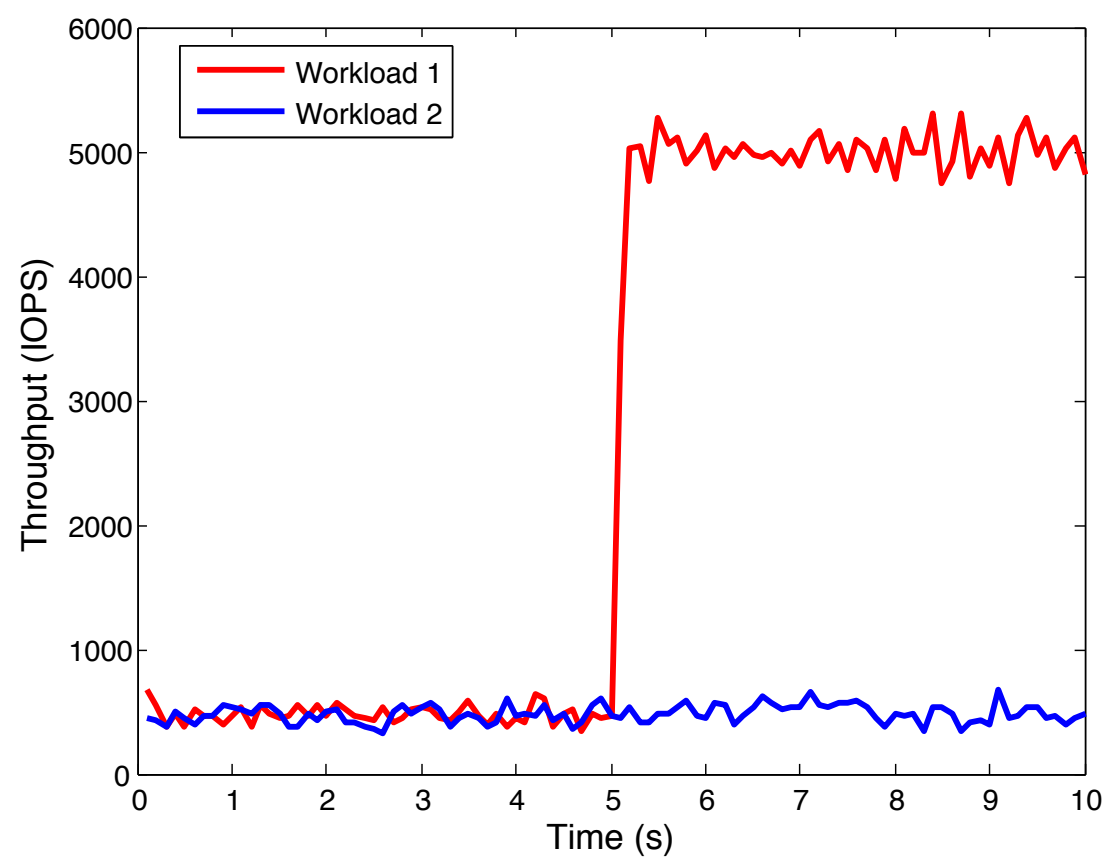

(a) Queue Length $=1$.

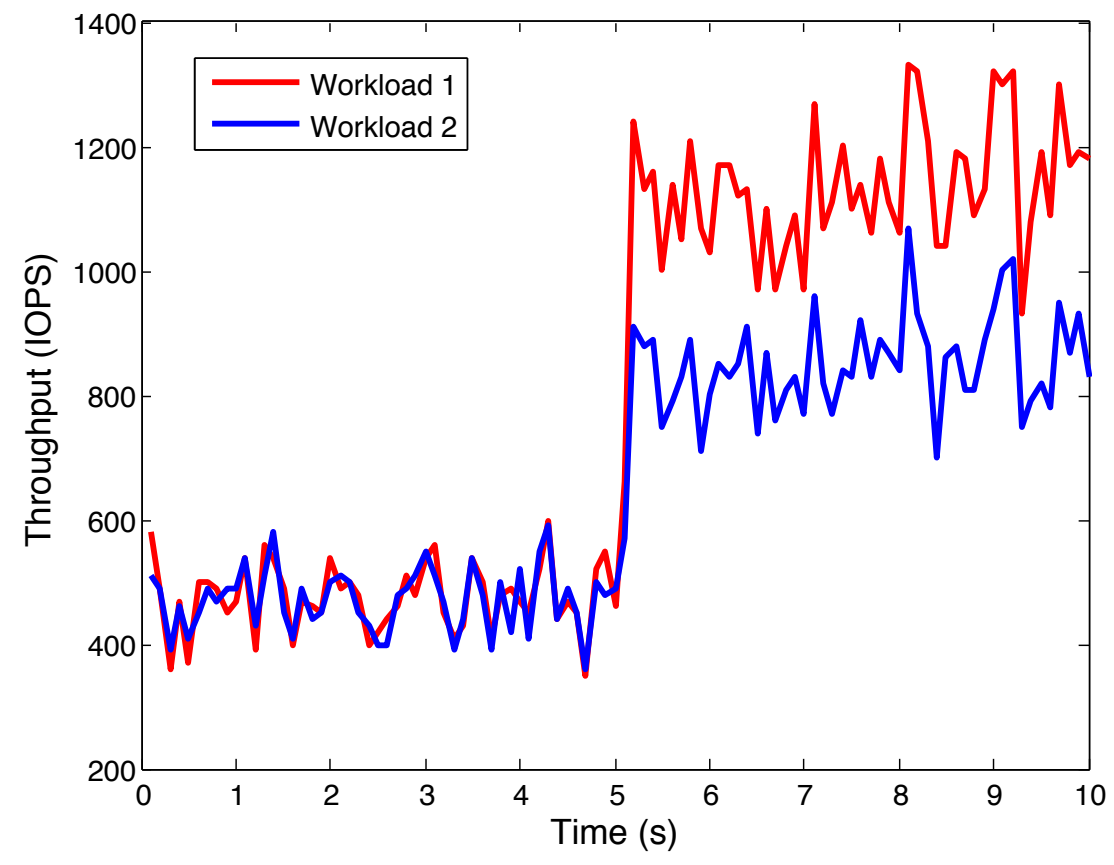

(b) Queue Length $=8$.

Figure 5.9 : Comparison of allocation when clients have different read/write ratios, with different queue lengths. 


\section{Chapter 6}

\section{Reservations and Limits}

In this chapter we consider an important practical extension to the basic reward model presented in Chapters 4 and 5, specifically the incorporation of reservations and limits. A reservation for client $i$, denoted by $R_{i}$, is a lower bound on IOPS guaranteed to it, while a limit, denoted by $L_{i}$ is an upper bound on the IOPS provided to it. Clients often like to place an upper-bound in a pay-for-service model to limit costs for low priority jobs or to prevent unexpected charges due to runaway applications. Reservations allow the client to anticipate a certain minimum performance under congested conditions; without reservations the client may receive very little allocation due to its low weight or because of a drop in available system capacity. We present the experimental evaluation of adding reservations to our allocation policy in Section 6.2.

In the reward model considered here, reservations are specially important for a client to protect itself from fluctuations in its own workload characteristics. A client may wish to reserve a minimum number of IOPS irrespective of its hit ratio. In this case, even if its entitlement falls due to a poor hit ratio, the reservation will ensure a floor. In this chapter we will focus on expanding the algorithm presented in Chapter 4 to add Reservations and Limits.

Handling Reservations: Guaranteeing reservations is difficult. The reward scheduler should ensure that each task gets at least its reservation while rewarding the tasks with lower service times. This requires that the system capacity must be sufficient to satisfy the reservations of all admitted clients, even under their worst-case 
access profiles (for instance, zero hit ratio). That is, admission control must ensure that the system capacity can't be less than a value denoted as $C_{m i n}$, which governed by Inequality 6.1 .

$$
C_{\min } \geq \sum_{j \in \mathcal{A}} R_{j}
$$

Other, more permissive admission control criteria can be adopted, by suitable Service Level Agreement (SLA) restrictions on the client workloads.

We handle reservations and limits using the elegant technique of using multiple tags proposed in [9]. Requests at the head of each queue are assigned a reservation tag (rTag) and a limit tag (lTag) in addition to the sTag described earlier. Successive rTags of client $i$ are equally spaced apart by $1 / R_{i}$. If a client's rTag lags the current time it means that it requires additional service to meet its reservation. If the rTag is ahead of the current time it means that all its reservations have been currently satisfied and it should be served according to its sTag. The sTags are updated as in Algorithm 1.

A client may dynamically change its access characteristics and move back and forth between being reservation bound or entitlement bound. In this case the scheduler needs to track the change, and synchronize between the rTags and sTags of the client. In [9] the two sets of tags were relatively independent and did not require any synchronization between them. Specifically, when a client switches from being reservation bound to entitlement bound (its hit ratio improves so that its weighted entitlement exceeds its reservation) its sTag needs to be resynchronized with the current rTag value. Otherwise it would face a prolonged delay before receiving its entitlement due to its sTag having run arbitrarily ahead of its rTag. We show this behavior experimentally in Section 6.2. The switch from being reservation bound to 
being entitlement bound is detected by checking if Inequality 6.2 is true or not.

$$
\frac{\Phi_{i}}{\omega_{i}}<\frac{1}{R_{i}}
$$

To prevent instability, we require this condition to hold over a certain minimum number of consecutive requests, to conclude that a genuine change of phase has occurred.

Handling Limits: The request at the head of the queue of client $i$ is assigned a limit $\operatorname{tag} \operatorname{lTag}_{i}$ that are spaced $1 / L_{i}$ apart. If the current time is less than $\mathbf{l T a g}_{i}$, it means that client $i$ has received more allocation than specified by its limit, and should not be serviced till $\operatorname{lTag}_{i}$ falls behind the current time.

\subsection{Reward Scheduler with Reservations and Limits}

The details of the algorithm extension are given in Algorithm 3. The scheduler searches the active client queues for requests whose $\mathbf{r T a g}$ are no more than the current time. The request with the smallest such reservation tag is selected for servicing and dispatched. If there are no eligible rTags, the scheduler selects the request with the lowest (sTag), provided its lTag is less than the current time. When a request completes, the service time $\Phi$ is recorded to be used in updating the average latency of the client, and for checking if there is a phase transition. 


\begin{tabular}{|c|l|}
\hline Symbol & Meaning \\
\hline \hline$L_{j}$ & Limit capacity of task $j$ \\
\hline$R_{j}$ & Reserved capacity of task $j$ \\
\hline $\mathbf{r} T a g_{j}$ & Reservation tag of task $j$ \\
\hline $\mathbf{l} T a g_{j}$ & Limit tag of task $j$ \\
\hline
\end{tabular}

Table 6.1 : Additional symbols used by the Reward Scheduler

The Reward Scheduling algorithm allocates the system's capacity in the following order: (1) Each client will get at least its reservation capacity provided the system capacity constraint is satisfied. This happens because the reservation tags have the highest priority in the system and requests required to satisfy a reservation are are always served in preference to other requests. (2) If there is additional capacity beyond that needed for reservations, it is allocated in accordance with the dynamic weights $\left(\bar{\Phi}_{i} / \omega_{i}\right)$ of the clients.

\subsection{Experimental Evaluation}

Finally we put all the pieces together and consider reward scheduling and reservations together.

Experiment 1: The only change from experiment 1 in Section 4.2.1 is that client $\mathrm{B}$ has a reservation of 60 IOPS. Hence during the initial interval $[0,25]$, client $\mathrm{B}$ must be allocated more than its entitlement of 50 IOPS; consequently the bandwidth allocated to clients $\mathrm{A}$ and $\mathrm{C}$ are reduced proportionately (see Figures 6.1(a) and 6.1(b)). At $t=25$ the hit ratio of client B changed to 0.4 .

We ran this experiment for two cases, The first is to show the starvation problem 


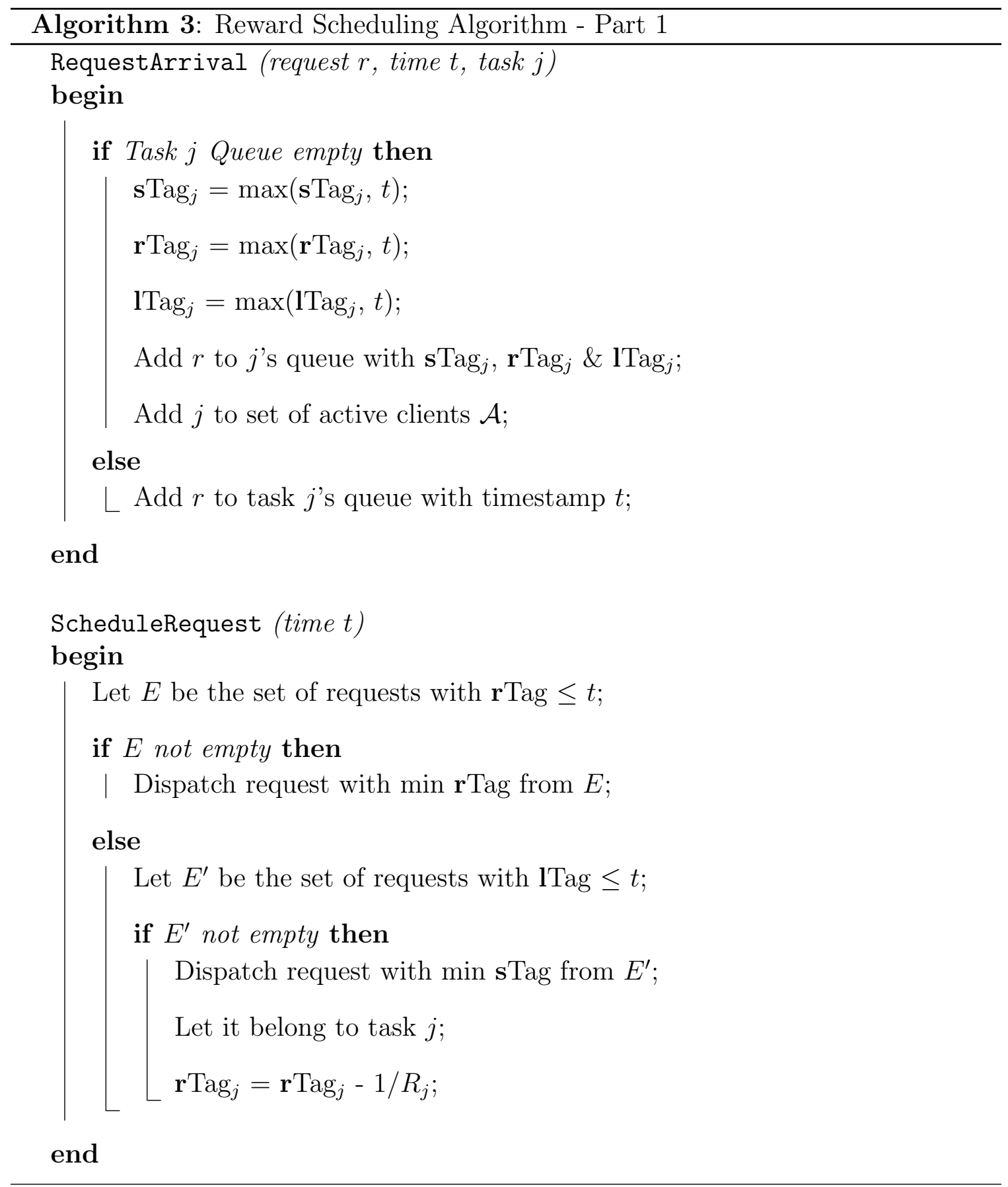




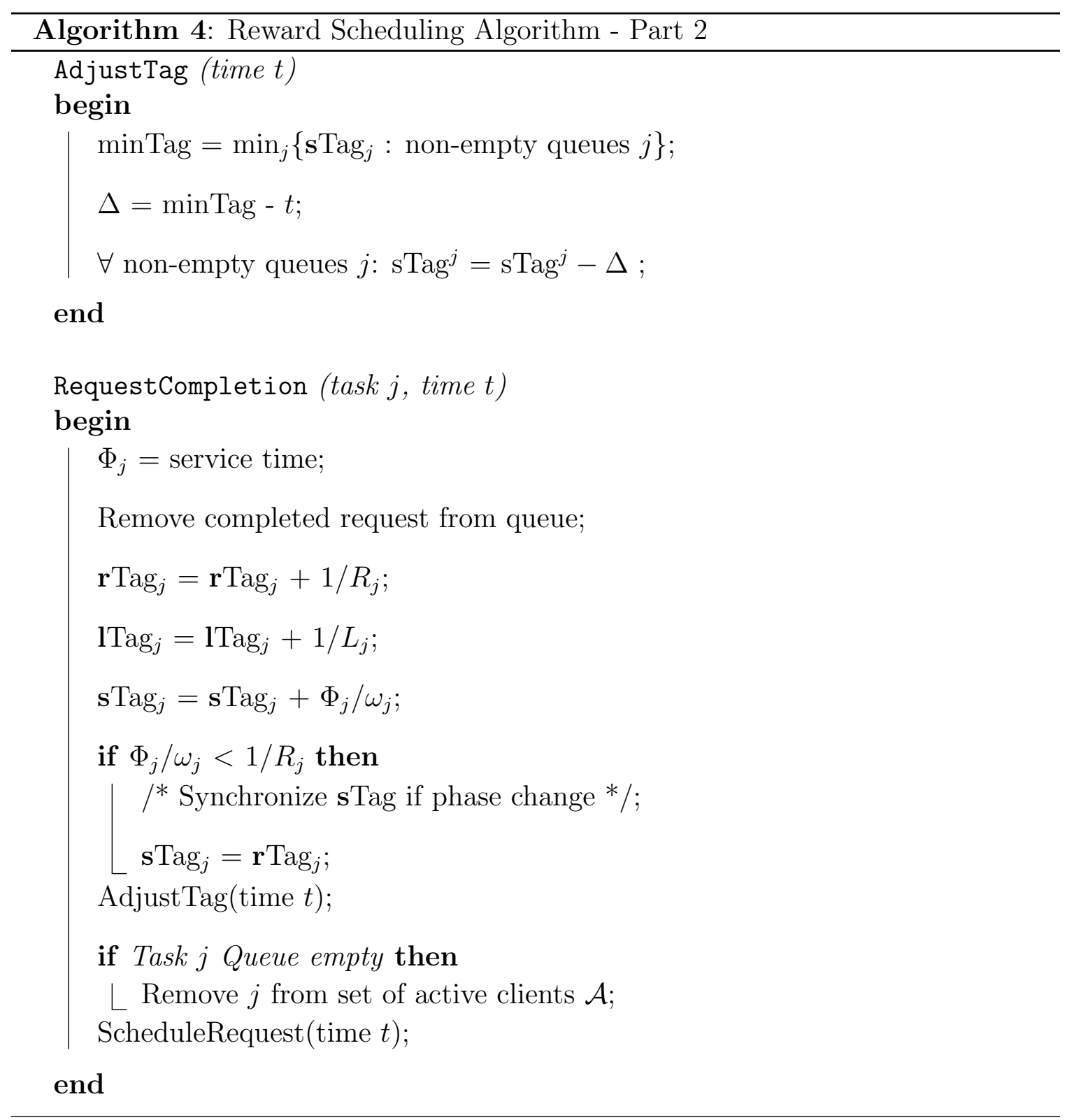


that arises if the sTags and rTags are not synchronized properly when the phase changes. As shown in Figure 6.1(a), the throughput allocated to B did not increase till roughly time 40 and it received additional bandwidth only for the interval $[40,75]$. The second, shown in Figure 6.1(b) where the tags are synchronized (Algorithm 3), client $\mathrm{B}$ receives additional bandwidth based on its entitlement almost immediately after its hit ratio changes, as desired. In Figure 6.1(b), the allocations of $\mathrm{A}$ and $\mathrm{C}$ are unaffected when the hit ratio changes. In Figure 6.1(a) the allocations to A and $\mathrm{C}$ increase during the interval $[25,40]$; during this time the system is generating additional IOs, but $\mathrm{B}$ is not receiving them due to the drift between its tags; hence the capacity is shared between $\mathrm{A}$ and $\mathrm{C}$ which see an increase in allocation in this interval.

Experiment 2: We expand to a multi-tiered server with one HD and one SSD, which can work in parallel. We use the same parameters as in Experiment 1, except that client B doesn't have any reservations and client A has a 40 IOPS reserved capacity. As can be seen from Figure 6.2, the scheduler successfully awarded client A the reserved capacity.

\subsection{Summary}

In this chapter, we expanded the reward allocation policy to support reservations and limits. Reservations and Limits are two desirable QoS parameters for clients and service providers. Reservations are useful for clients to insure a minimum level of service, while limits are useful for service providers to prevent one client from getting all the system's capacity. 


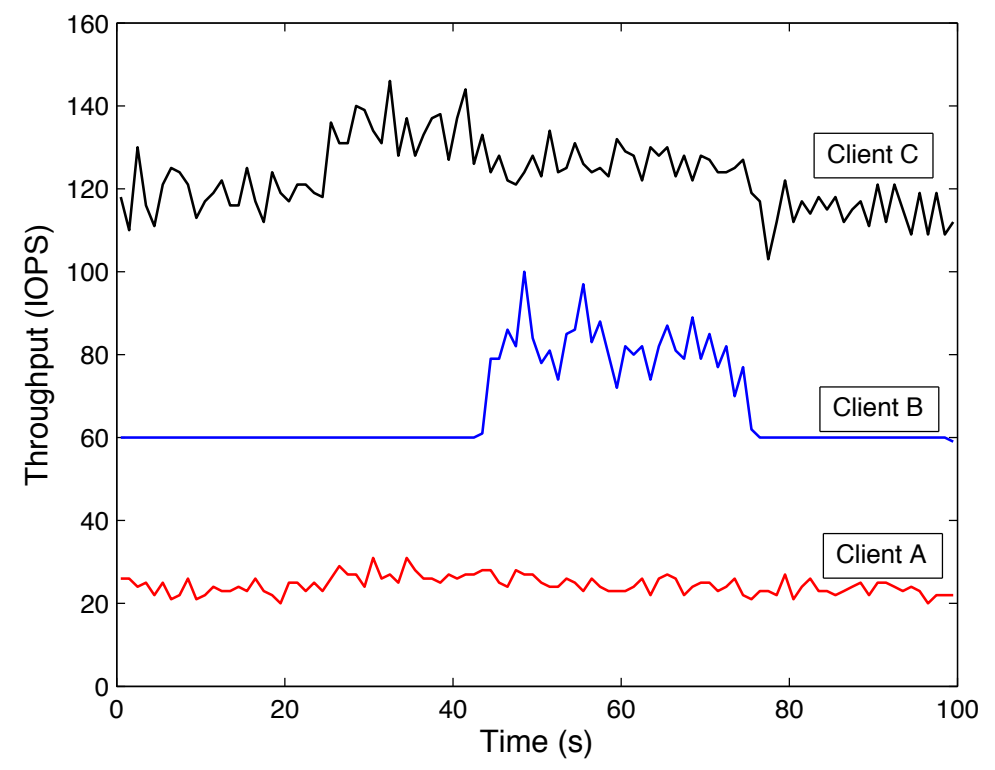

(a) Starvation in Reservation

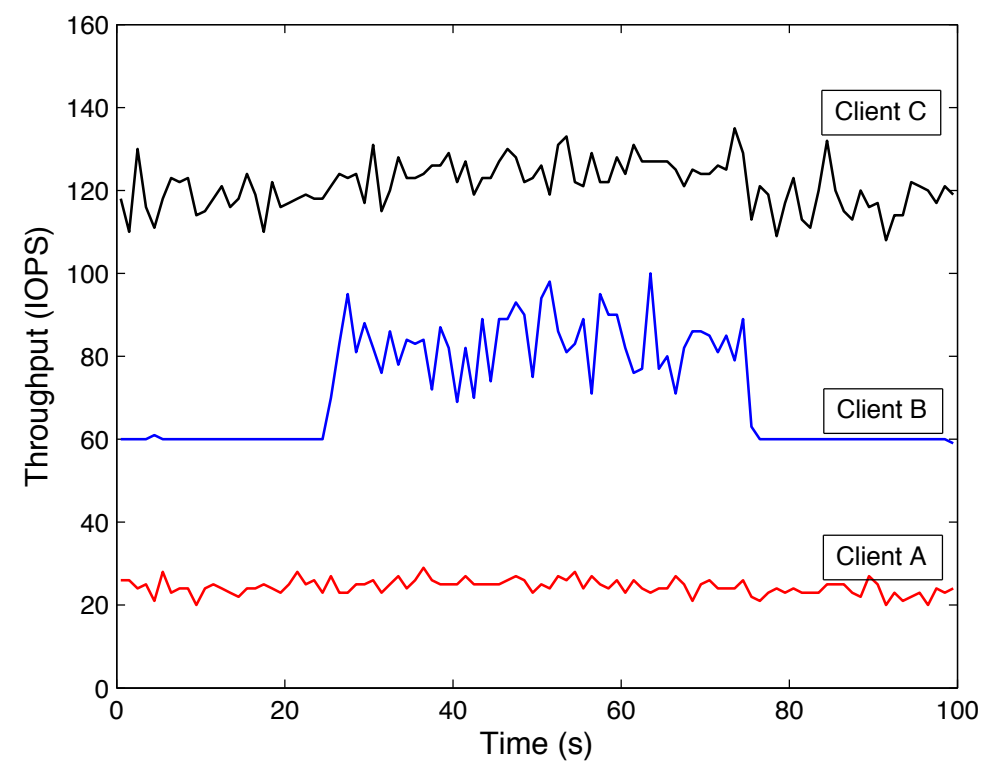

(b) Reward Scheduling with Reservations

Figure 6.1 : Comparison Between Capacity Allocation With and Without rTag/wTag Synchronization 


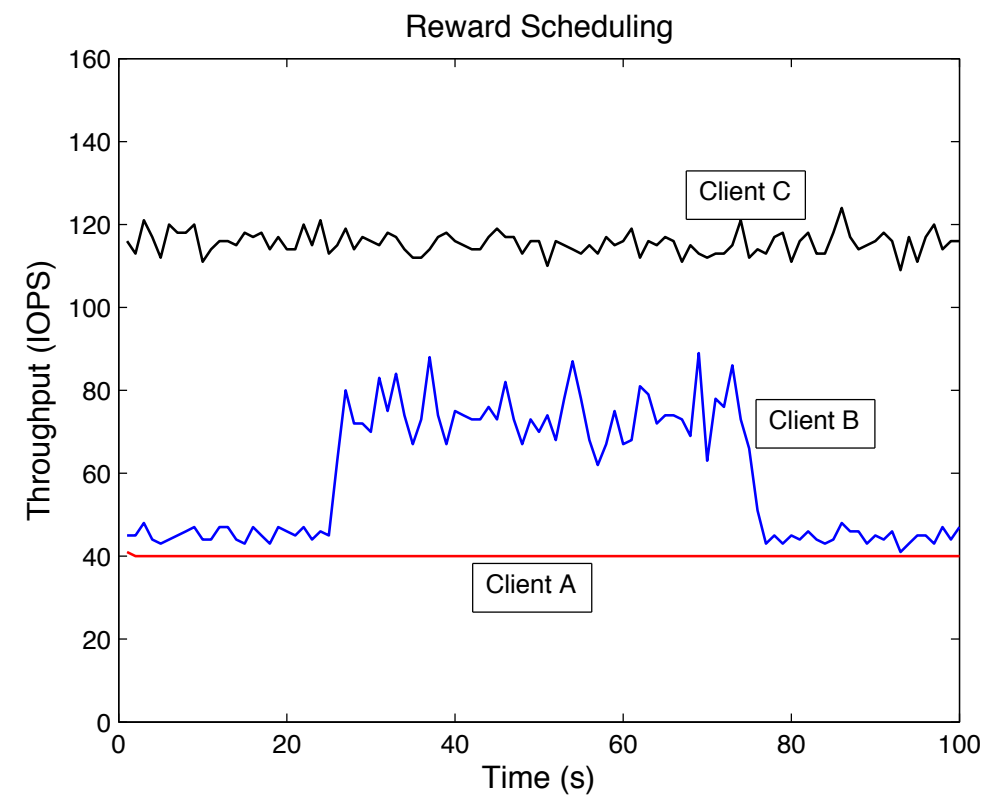

Figure 6.2 : Reward Scheduling with Reservations in Parallel Model 


\section{Chapter 7}

\section{Conclusion}

In this thesis, we presented a novel QoS scheduling algorithm for multi-tiered storage servers made up of hard disks and SSDs. Our scheme is designed to rewards clients according to their runtime behavior, while honoring their static QoS settings including shares (or weights), reservations and limits. The work is motivated by the difference in access times and hit ratios for a workload in different phases of execution as its hit ratio to the SSD device changes. A model based on entitlements is developed to describe the reward allocation policy. Simulation results show the advantages of this policy over conventional proportional share allocation, and its ability to adapt to dynamically varying workloads.

We believe that the proposed reward scheduling algorithm leads to QoS guarantees, which are more useful to clients than existing proportional share schemes in multi-tenant shared environments. The proposed algorithm allows the client to directly reap the benefits of application performance tuning as if the client is on a dedicated system. This addresses a key complaint of clients when moving to a shared infrastructure. From the infrastructure's viewpoint, reward scheduling also results in higher throughput. 


\section{Bibliography}

[1] R. N. Calheiros, C. Vecchiola, D. Karunamoorthya, and R. Buyyaa, "The Aneka platform and QoS-driven resource provisioning for elastic applications on hybrid clouds," Future Generation Computer Systems, 2011.

[2] "VMWare vSphere 5: Private Cloud Computing, Server and Data Center Virtualization." http://www.vmware.com/products/vsphere/overview.html, 2011.

[3] "Microsoft Server and Cloud Platform." http://www.microsoft.com/enus/server-cloud/windows-server/hyper-v.aspx, 2011.

[4] "XenServer6." http://www.citrix.com/English/ps2/products/product.asp? contentID $=683148,2011$.

[5] "Amazon Web Services." http://aws.amazon.com, 2011.

[6] "Bluelock." http://www.bluelock.com/, 2011.

[7] "Verizon Cloud." http://www.verizonbusiness.com/Medium/products/ itinfrastructure/computing/, 2011.

[8] A. Gulati, I. Ahmad, and C. Waldspurger, "PARDA: Proportional Allocation of Resources for Distributed Storage Access," in In FAST '09: Proceedings of the 7th Usenix Conference on File and Storage Technologies, pp. 85-98, 2009.

[9] A. Gulati, A. Merchant, and P. J. Varman, "mClock: Handling throughput variability for hypervisor IO scheduling," in USENIX OSDI, pp. 1-7, 2010. 
[10] P. Manning, "Storage i/o control technical overview and considerations for deployment," VMWare White Paper, 2010.

[11] "Nutanix Complete Cluster: The New Virtualized Datacenter Building Block." http://www.nutanix.com/resources.html, 2011.

[12] A. Elnably, K. Du, and P. Varman, "Reward Scheduling for QoS in Cloud Applications," in 12th IEEE/ACM International Symposium on Cluster, Cloud, and Grid Computing, May 2012 (to appear).

[13] A. Elnably and P. Varman, "Application-Sensitive QoS Scheduling in Storage Servers," in ACM Symposium on Parallelism in Algorithms and Architecture, June 2012 (short paper to appear).

[14] A. Elnably, H. Wang, A. Gulati, and P. Varman, "Efficient QoS for Multi-Tiered Storage Systems," in 4th USENIX Workshop on Hot Topics in Storage and File Systems, 2012 (to appear).

[15] A. Demers, S. Keshav, and S. Shenker, "Analysis and simulation of a FairQueuing algorithm," in ACM SIGCOMM, pp. 1-12, 1989.

[16] C. A. Waldspurger and W. E. Weihl, "Lottery scheduling: flexible proportionalshare resource management," in Proceedings of the 1st USENIX Conference on Operating Systems Design and Implementation, 1994.

[17] P. Goyal, H. M. Vin, and H. Chen, "Start-time fair queueing: a scheduling algorithm for integrated services packet switching networks," in Proceedings on Applications, Technologies, Architectures, and Protocols for Computer Communications, pp. 157-168, 1996. 
[18] P. J. Shenoy and H. M. Vin, "Cello: a disk scheduling framework for next generation operating systems," in Proceedings of the 1998 ACM SIGMETRICS joint international conference on Measurement and modeling of computer systems, pp. 44-55, 1998.

[19] R. Wijayaratne and A. L. N. Reddy in Proceedings of the IEEE International Conference on Multimedia Computing and Systems.

[20] C. R. Lumb, J. Schindler, G. R. Ganger, D. F. Nagle, and E. Riedel, "Towards higher disk head utilization: extracting free bandwidth from busy disk drives," in Proceedings of the 4 th Conference on Symposium on Operating System Design 6 Implementation, p. 7, 2000.

[21] C. R. Lumb, J. Schindler, and G. R. Ganger, "Freeblock scheduling outside of disk firmware," in Proceedings of the 1st USENIX Conference on File and Storage Technologies, p. 20, 2002.

[22] W. Jin, J. S. Chase, and J. Kaur, "Interposed proportional sharing for a storage service utility," in Proceedings of the Joint International Conference on Measurement and Modeling of Computer Systems, pp. 37-48, 2004.

[23] J. Zhang, A. Subramaniam, Q. Wang, A. Riska, and E. Riedel, "Storage performance virtualization via throughput and latency control," Trans. Storage, vol. 2, pp. 283-308, August 2006.

[24] A. Gulati, A. Merchant, and P. J. Varman, "pClock: An arrival curve based approach for QoS in shared storage systems," in Proceedings of the 2007 ACM SIGMETRICS international conference on Measurement and modeling of computer systems, pp. 13-24, 2007. 
[25] H. Chang, R. Chang, W. Shih, and R. Chang, "Gsr: A global seek-optimizing real-time disk-scheduling algorithm," Journal of Systems and Software, vol. 80, no. 2, pp. 198-215, 2007.

[26] L. L. Abeni, G., and G. Buttazzo, "Constant bandwidth vs. proportional share resource allocation," in IEEE International Conference on Multimedia Computing and Systems, vol. 2, pp. 107-111, 1999.

[27] D. J. Shakshober, "Choosing an I/O Scheduler for Red Hat Enterprise Linux 4 and the 2.6 Kernel," in In Red Hat magazine, June 2005.

[28] S. Park and K. Shen, "FIOS: A Fair, Efficient Flash I/O Scheduler," in FAST, 2012.

[29] A. Povzner, T. Kaldewey, S. Brandt, R. Golding, T. M. Wong, and C. Maltzahn, "Efficient guaranteed disk request scheduling with fahrrad," in Proceedings of the 3rd ACM SIGOPS/EuroSys European Conference on Computer Systems, pp. 1325, 2008.

[30] P. Valente and F. Checconi, "High Throughput Disk Scheduling with Fair Bandwidth Distribution," in IEEE Transactions on Computers, no. 9, pp. 1172-1186, 2010.

[31] M. Wachs, M. Abd-El-Malek, E. Thereska, and G. Ganger, "Argon:Performance Insulation for Shared Storage Servers," in In FAST 'O'\%: Proceedings of the 5th Usenix Conference on File and Storage Technologies, pp. 61-76, 2007.

[32] J. Bruno, J. Brustoloni, E. Gabber, B. Ozden, and A. Silberschatz, "Disk scheduling with quality of service guarantees," in Proceedings of the IEEE International Conference on Multimedia Computing and Systems, pp. 400-405, 1999. 
[33] M. Karlsson, C. Karamanolis, and X. Zhu, "Triage: Performance differentiation for storage systems using adaptive control," Trans. Storage, vol. 1, pp. 457-480, 2005.

[34] T. Wong, R. Goldering, C. Lin, and R. Becker-Szendy, "Zygaria: Storage performance as managed resource," in Proc. of RTAS, pp. 125-34, April 2006.

[35] D. Gupta, L. Cherkasova, R. Gardner, and A. Vahdat, "Enforcing performance isolation across virtual machines in Xen," in Proceedings of the ACM/IFIP/USENIX 2006 International Conference on Middleware, pp. 342$362,2006$.

[36] J. R. Jump, "Yacsim reference manual." http://oucsace.cs.ohiou.edu/ avinashk/ classes/ee690/yac.ps. 